\title{
Linear damped interfacial wave theory for an orbitally shaken upright circular cylinder
}

\author{
G. M. Horstmann ${ }^{1, \dagger}$, W. Herreman ${ }^{2}$ and T. Weier ${ }^{1}$ \\ ${ }^{1}$ Institute of Fluid Dynamics, Helmholtz-Zentrum Dresden - Rossendorf, Bautzner Landstr. 400, \\ 01328 Dresden, Germany \\ ${ }^{2}$ LIMSI, CNRS, Université Paris-Sud, Université Paris-Saclay, Orsay, F-91405, France
}

(Received 4 March 2019; revised 29 January 2020; accepted 25 February 2020)

We present a new theoretical model describing gravity-capillary waves in orbitally shaken cylindrical containers. Our model can account for both one-layer free-surface and two-layer interfacial wave systems. A set of modal equations for irrotational waves is formulated that we complement with viscous damping rates to incorporate energy dissipation. This approach allows us to calculate explicit formulas for the phase shifts between wave and shaker which are practically important for the mixing efficiency in orbitally shaken bioreactors. Resonance dynamics is described using eight dimensionless numbers, revealing a variety of different effects influencing the forced wave amplitudes. As an unexpected result, the model predicts the formation of novel spiral wave patterns resulting from a damping-induced symmetry breaking mechanism. For validation, we compare theoretical amplitudes, fluid velocities and phase shifts with three different and independent experiments and, when using the slightly deviating experimental values of the resonance frequencies, find a good agreement within the theoretical limits.

Key words: wave-structure interactions

\section{Introduction}

Orbital sloshing experiments provide the opportunity to study a broad range of physical effects in the field of fluid mechanics with moderate effort. An orbital shaking device can perform circular trajectories with adjustable shaking diameters $d_{s}$ and angular frequencies $\Omega$. This motion naturally induces a homogeneous, rotating centripetal force, which can be exploited to drive rotating gravity or capillary waves accompanied by a swirling mean flow in partially filled cylindrical tanks.

Although such experiments might appear unsophisticated, we know today that the evolving wave dynamics can be quite complex. Multiple linear and nonlinear, harmonic and sub-harmonic, synchronous and non-synchronous wave modes may arise. These modes can involve linear or nonlinear resonance dynamics subject to a hysteresis or even wave breaking. Different damping mechanisms require a profound

$†$ Email address for correspondence: g.horstmann@hzdr.de 
understanding of the viscous boundary layers. Additionally, the wave-induced flow fields can comprise different secondary flow structures as well as a swirling mean flow. Finally, capillary effects at the surface-sidewall contact line can modify the entire wave dynamics or cause additional damping. In addition to these basic research topics, there is a substantial amount of applied research on orbitally shaken bioreactors (OSBs), the most important current application. In the following, we will give a short overview of orbital sloshing research.

The first known orbital sloshing apparatus was realised by Ludwig Prandtl around 1940, who intended to understand the origin of the mean swirling flow (Prandtl 1949; Eckert 2019), a question which is still the subject of research. Mainly motivated by spacecraft and offshore applications, sloshing dynamics research in the 1950s concentrated on hydrodynamic loads in moving containers under lateral, rolling or pitching excitation; see Abramson (1966). In contrast, orbital sloshing, which is less connected to these practical applications, remained almost unexplored with the exception of Hutton (1964). Hutton developed analytical expressions for the velocity field and the surface elevation by applying potential flow theory.

Orbitally excited hydrodynamics came into focus only much later at the beginning of the 21 st century due to the growing interest in orbitally shaken bioreactors. Modern OSBs can provide mixing, aeration and shear stresses on multiple scales and are meanwhile used for various applications such as the cultivation of mammalian stem cells, drug production or fermentation processes (Klöckner \& Büchs 2012; Klöckner, Diederichs \& Büchs 2014). While earlier studies had focused mainly on the gas exchange, mixing or the power consumption (Büchs et al. 2000; Micheletti et al. 2006; Zhang et al. 2009; Tissot et al. 2010; Klöckner et al. 2012), research into wave and fluid mechanics has gained importance in recent years. Kim \& Kizito (2009) were the first to experimentally and numerically study the wave-induced flow fields and found that the swirling mean flow is mainly driven by secondary flow structures.

Significant experimental progress was then achieved by Weheliye, Yianneskis \& Ducci (2013) and Ducci \& Weheliye (2014), who measured velocity fields for a wide range of parameter combinations. From these measurements they could deduce useful scaling laws to predict the interface elevation, they managed to characterise different flow regimes and, perhaps most importantly, they could characterise the transition to the out-of-phase flow regime that is essential for the bioreactor efficiency in terms of mixing times (Rodriguez et al. 2014), microcarrier suspension speeds (Pieralisi et al. 2016) or power consumption (Büchs et al. 2000). Complementary, studies by Reclari (2013) and Reclari et al. (2014) investigated in detail the wave dynamics. Using inviscid potential flow theory, they developed a weakly nonlinear model to predict the wave motion and velocity fields. This model is still state-of-the-art and can characterise and classify many different wave patterns. However, the wave dynamics under close-to-resonance excitation is out of the scope of this model. There, it diverges, and waves behave strongly nonlinearly under typical shaking conditions. This gap was recently filled by Timokha \& Raynovskyy (2017) and Raynovskyy \& Timokha (2018b) who have applied the Narimanov-Moiseev multimodal sloshing theory describing the nonlinear wave dynamics near the first resonance. They have proven that resonant wave dynamics is of the hard-spring type and could explain its frequency-dependent hysteresis, observed before by Reclari (2013). Also Prandtl's original question about the origin of the mean swirling flow slid back into focus. Bouvard, Herreman \& Moisy (2017) have studied the Lagrangian mean flow in the weakly nonlinear regime and attributed the mean central rotation primarily to 
the Stokes drift. Thereafter, Faltinsen \& Timokha (2019) provided a comprehensive theoretical description of both the Eulerian and non-Eulerian mean azimuthal mass transport, pointing out that the Stokes drift can explain the mass transport only in a small neighbourhood around the tank centre. Moisy, Bouvard \& Herreman (2018) have investigated the mean flow under the influence of a thin layer of foam and explained the formation of a counter-rotating flow. Beside these fundamental approaches, there is currently ongoing research on different flow properties of practical relevance for the design and optimisation of OSBs: wall shear stresses, volumetric mass transfer, mixing times or wave breaking regimes (Discacciati et al. 2013; Rodriguez et al. 2013; Filipovic et al. 2016; Pieralisi et al. 2016; Thomas et al. 2017; Alpresa et al. 2018a,b; Rodriguez, Micheletti \& Ducci 2018; Weheliye et al. 2018; Zhu et al. 2018).

A related magnetohydrodynamic question stimulated our own interest in the topic. Horstmann, Wylega \& Weier (2019) have introduced a novel multi-layer interfacial sloshing wave experiment, which is physically related to the presented free-surface sloshing studies. It was designed with the intention to imitate the responding wave dynamics, as it can be induced by the magnetohydrodynamical metal pad roll instability, which is a potential limiting factor in aluminium reduction cells and liquid metal batteries (Bojarevics \& Romerio 1994; Weber et al. 2017; Horstmann, Weber \& Weier 2018; Kelley \& Weier 2018; Tucs, Bojarevics \& Pericleous 2018).

It became apparent that understanding of the effects of viscous damping on resonance dynamics, particularly for the case of interfacial waves, can be improved. The frequently applied potential model of Reclari et al. (2014) fails to predict the wave amplitudes near resonance, the most interesting regime, due to the lack of any dissipation source term. Likewise, a satisfactory explanation of the overdamped resonance amplitudes, described by Horstmann et al. (2019), is lacking so far. Also, the phase lags between shaker and wave are not yet quantitatively understood. There are at least two different physical mechanisms which are frequently confused: on the one hand, we have the classical phase jumps of $180^{\circ}$ emerging around the resonance frequencies, which are smoothed out by damping forces. These kinds of phase shifts are, inter alia, discussed by Reclari et al. (2014), Bouvard et al. (2017), Alpresa et al. (2018a) and Horstmann et al. (2019). We will refer to them henceforth as linear phase shifts, although they also appear in the weakly nonlinear regime, where they can be subject to a hysteresis (Raynovskyy \& Timokha 2018b). On the other hand, Weheliye et al. (2013), Klöckner et al. (2014), Ducci \& Weheliye (2014) and Weheliye et al. (2018) investigate strongly nonlinear phase shifts, which are induced by the interaction of secondary flow structures with the vessel walls. These phase shifts are essential for the mixing efficiency (Rodriguez et al. 2014) and can arise long before the first resonance is reached.

In the paper at hand we develop a new damped sloshing model accounting for both gravity-capillary free-surface and interfacial waves in cylindrical containers. Consequently, the model can be applied to better understand wave dynamics in many OSB as well as to study damping mechanisms in multi-layer stratifications such as liquid metal batteries. Aiming for explicit solutions, we derive at first a set of modal equations accounting for the irrotational part of the flow only and include linear damping rates a posteriori. Viscous damping rates of interfacial waves in cylinders were recently derived by Herreman et al. (2019) and are applied for the two-layer description. Analogous damping rates of free-surface waves are well known (Miles \& Henderson 1998) and have already been included in the nonlinear resonant sloshing theory of Raynovskyy \& Timokha (2018b). Our analysis is restricted only to linear 
solutions since we found that they already capture several of the aforementioned phenomena and allow for some predictions in the weakly nonlinear regime as well.

The paper is organised as follows. In $\S 2$, we formulate the sloshing problem and derive a set of modal equations, which is supplemented by linear damping rates. We present explicit solutions for the wave amplitudes and phase shifts which depend on eight independent dimensionless numbers. In $\S 3$, we discuss the resulting wave and resonance dynamics for the different cases considered. As a special feature, the theory predicts the occurrence of novel spiral wave patterns, which are analysed for various damping rates. In $\S 4$, we finally compare theoretical wave amplitudes, phase shifts and fluid velocities with three recently reported experiments. Section 4.3 contains an explanation of the wave elevation's linear scaling with the Froude number that was found by Weheliye et al. (2013) on an empirical basis. Building on this, a prediction for the nonlinear out-of-phase transition is also derived by quantifying the waviness of the free surface.

\section{Theory}

Analytical solutions to viscous wave dynamics are often difficult if not impossible to find. Therefore, potential flow theory was frequently applied in many early studies to approximate the velocity fields. However, potential flow theory introduces drastic simplifications: the flow is assumed to be inviscid, irrotational and incompressible. Further, wave amplitudes must remain sufficiently low to allow linear or weakly nonlinear approaches. Despite all these restrictions, potential approaches have been quite successful in predicting non-resonant sloshing (Ibrahim 2005).

Studies tackling viscous wave theories and their higher mathematical complexity are rare. A significant approach for lateral excitation was developed by Bauer \& Eidel (1997, 1999), who performed a modal analysis starting from the Stokes equations. This approach allows us to calculate responding resonance curves and container forces in direct dependence of the viscosity; however, the solutions are elusive and remain implicit. For this reason, we decided to apply a simpler approach based on potential flow theory. Although potential flow theory can be applied only to irrotational flows, it is possible to include viscous damping rates a posteriori into the modal equations, as described by Faltinsen \& Timokha (2009, chap. 6). In this way, we neglect the influence of rotational boundary layers on the flow fields and the wave motion, but we can include the energy dissipation resulting from the boundary layers. This approach is valid only if the boundary layers are considerably smaller than the lateral dimensions of the cylinder. The detailed parameter regime fulfilling this condition is discussed in $\S 2.5$.

Very recently, Herreman et al. (2019) have derived viscous damping rates for interfacial waves in cylindrical tanks using a perturbative description of Stokes boundary layers. This result finally allows us to derive explicit viscosity-dependent formulas for the resonance curves and phase shifts, which are comparatively easy to handle and simple to apply for further analysis.

\subsection{Statement of the problem}

Figure 1 shows the set-up for our theoretical framework, analogous to the description given by Reclari (2013). We define an ideal cylinder of radius $R$, containing two liquid layers (subscripts $i=1,2$ ) of heights $h_{1}, h_{2}$, kinematic viscosities $v_{1}, v_{2}$ and densities $\rho_{1}, \rho_{2}$, where $\rho_{1}<\rho_{2}$ must be fulfilled to ensure a stable vertical stratification. Gravity $\boldsymbol{g}$ acts in the negative $z$-direction. In cylindrical coordinates $(r, \varphi, z)$ the interface is 


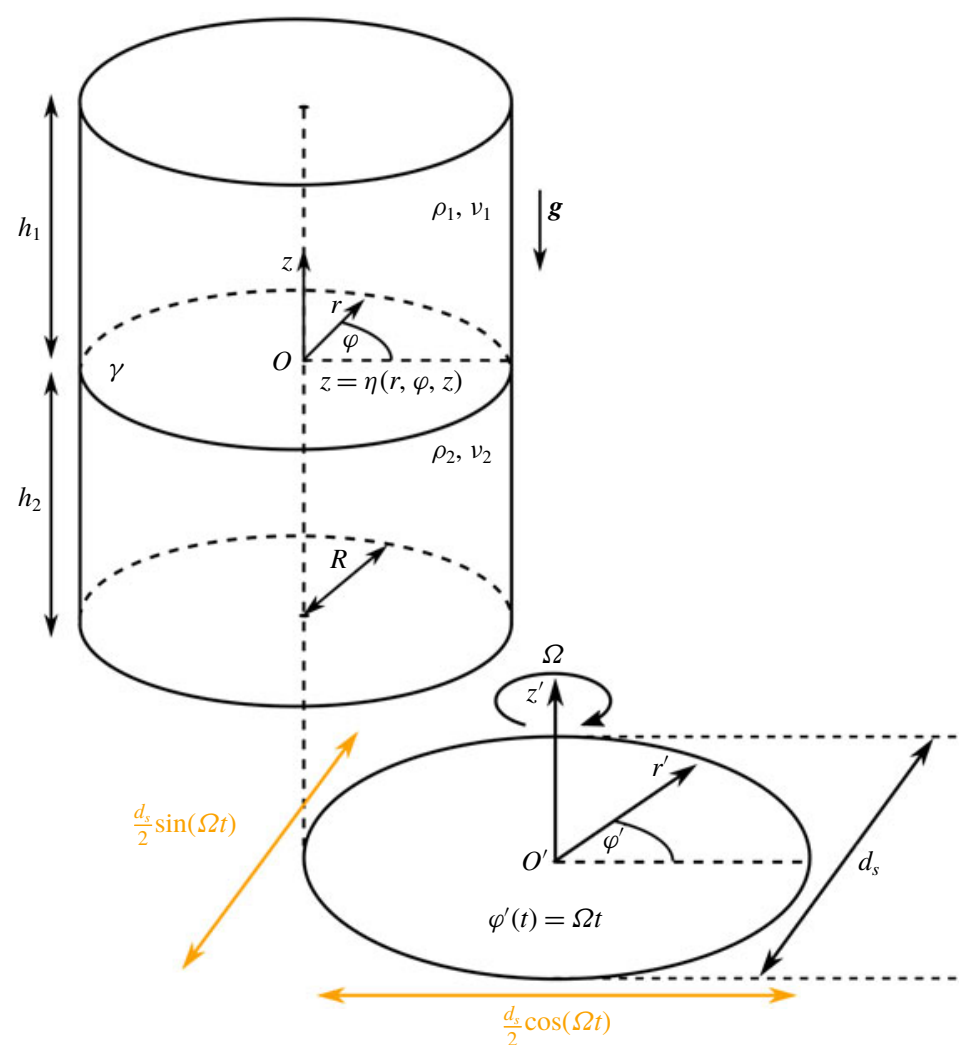

FIGURE 1. Sketch of the theoretical set-up. A cylinder of radius $R$ undergoes a harmonic circular translation of diameter $d_{s}$ with a constant angular frequency $\Omega$. The cylinder contains two fluids $i=1,2$ of densities $\rho_{i}$, kinematic viscosities $v_{i}$ and layer heights $h_{i}$, which are stably stratified due to gravity $\boldsymbol{g}$. The interface with interfacial tension $\gamma$ is placed at $z=\eta(r, \varphi, t)$ in the frame of reference moving with the cylinder $O(r, \varphi, z)$. The inertial frame of reference is indexed by $O^{\prime}\left(r^{\prime}, \varphi^{\prime}, z^{\prime}\right)$. The orange arrows mark the decompositions of the orbital translation into Cartesian coordinates $\left(\boldsymbol{e}_{x}, \boldsymbol{e}_{y}\right)$.

located at $z=\eta(r, \varphi, t)$. Interfacial tension $\gamma$ is considered along the liquid-liquid interface but capillary forces acting on the contact line are neglected; we assume that the interface may slide freely along the cylinder wall while maintaining a static contact angle of $90^{\circ}$ (no meniscus). The coordinate origin of the moving cylinder, the moving frame of reference $O(r, \varphi, z)$, is defined in the centre of the unperturbed interface $\eta$. In addition, we define the inertial frame of reference $O^{\prime}\left(r^{\prime}, \varphi^{\prime}, z^{\prime}\right)$ to describe the circulatory trajectories of the shaking table. The shaker is horizontally translated with a constant angular frequency $\Omega$ along a circular path $\varphi^{\prime}(t)=\Omega t$ of shaking diameter $d_{s}$.

This formulation is characterised by eleven physical variables and three physical dimensions. Following the Buckingham $\pi$ theorem, the system can be uniquely described by eight independent dimensionless parameters. We define the following set of dimensionless numbers for our analysis:

$$
F r=\frac{d_{s} \Omega^{2}}{2 g}, \quad E=\frac{d_{s}}{2 R}, \quad R e_{i}=\frac{\Omega R^{2}}{v_{i}}, \quad H_{i}=\frac{h_{i}}{R}, \quad B o=\frac{\left(\rho_{2}-\rho_{1}\right) g R^{2}}{\gamma}, \quad A=\frac{\rho_{2}-\rho_{1}}{\rho_{1}+\rho_{2}}
$$


The Froude number $F r$ describes the forcing expressed by the ratio of inertial force and gravitational acceleration. The normalised shaking diameter determines the eccentricity $E$, while $R e_{i}$ are the phase-dependent Reynolds numbers, here weighting the cylinder radius with the boundary layer thicknesses $\delta_{i}=\sqrt{v_{i} / \Omega}$. The importance of gravitational forces compared with interfacial tension forces is specified by the Bond number $B o$. Finally, $H_{i}$ and $A$ are the geometrical aspect ratios and the Atwood number, respectively. It is the aim of our following analysis to relate these numbers to the resulting wave amplitudes and phase shifts.

\subsection{Modal equations and solutions}

The velocity vector $\boldsymbol{V}_{0}(t)$ describing the orbital container motion can be expressed in terms of cylindrical unit base vectors in the inertial frame of reference $O^{\prime}\left(r^{\prime}, \varphi^{\prime}, z^{\prime}\right)$

$$
\boldsymbol{V}_{0}(t)=\left(\begin{array}{c}
-\frac{d_{s} \Omega}{2} \sin \left(\Omega t-\varphi^{\prime}\right) \boldsymbol{e}_{r^{\prime}} \\
\frac{d_{s} \Omega}{2} \cos \left(\Omega t-\varphi^{\prime}\right) \boldsymbol{e}_{\varphi^{\prime}}
\end{array}\right)
$$

Due to this orbital motion the liquids in the cylinder constantly experience rotary acceleration in the moving frame of reference $O$ resulting in centripetal forces, which can be formulated as external volume forces in the equations of motion for the container-fixed coordinate system. The incompressible Euler equations for $O$ under the influence of gravity $\boldsymbol{g}$ and subject to $\boldsymbol{V}_{0}$ are

$$
\begin{gathered}
\frac{\partial \boldsymbol{u}_{i}}{\partial t}+\frac{1}{2} \nabla\left|\boldsymbol{u}_{i}^{2}\right|-\boldsymbol{u}_{i} \times\left(\nabla \times \boldsymbol{u}_{i}\right)+\frac{1}{\rho_{i}} \nabla P_{i}-\boldsymbol{g}+\dot{\boldsymbol{V}}_{0}=0, \\
\nabla \cdot \boldsymbol{u}_{i}=0,
\end{gathered}
$$

(compare with Kochin, Kibel \& Roze (1964, chap. 2)), where $\boldsymbol{u}_{i}$ are the relative velocity fields and $P_{i}$ the pressures associated with both fluids $(i=1,2)$. By postulating irrotationality $\left(\nabla \times \boldsymbol{u}_{i}=0\right)$, the potential flow approximation can be applied stating that the flow fields $\boldsymbol{u}_{i}$ can be uniquely expressed as gradients of scalar potentials $\phi_{i}$

$$
\boldsymbol{u}_{i}=\nabla \phi_{i}
$$

By inserting (2.5) into (2.3) and (2.4) and integrating over the cylinder volume, a complete set of governing equations and boundary conditions for the scalar flow potentials $\phi_{i}$, the pressures $P_{i}$ and the interface position $\eta$ can be formulated

Flow fields: $\quad \frac{\partial \phi_{i}}{\partial t}-\frac{d_{s} \Omega^{2} r}{2} \cos (\Omega t-\varphi)+\frac{1}{2}\left(\nabla \phi_{i}\right)^{2}+\frac{P_{i}}{\rho_{i}}+g z=c_{i}(t)$,

Flow fields: $\quad \Delta \phi_{i}=0$,

$$
\text { Top wall: } \quad \frac{\partial \phi_{1}}{\partial z}=\left.0\right|_{z=h_{1}},
$$

Bottom wall: $\quad \frac{\partial \phi_{2}}{\partial z}=\left.0\right|_{z=-h_{2}}$,

Sidewall: $\quad \frac{\partial \phi_{1}}{\partial r}=\frac{\partial \phi_{2}}{\partial r}=\left.0\right|_{r=R}$, 


$$
\begin{array}{ll}
\text { Interface: } & \frac{\partial \phi_{1}}{\partial z}=\frac{\partial \phi_{2}}{\partial z}=\frac{\partial \eta}{\partial t}+\left.\nabla \phi_{i} \cdot \nabla \eta\right|_{z=\eta}, \\
\text { Interface: } & \gamma \nabla \cdot \frac{\nabla \eta}{\sqrt{1+|\nabla \eta|^{2}}}=P_{1}-\left.P_{2}\right|_{z=\eta} .
\end{array}
$$

The first two equations (2.6a) and (2.6b) are the instationary Bernoulli equation and the Laplace equation ensuring energy and mass conservation. Equations $(2.6 c),(2.6 d)$ and (2.6e) comprise the kinematic no-outflow boundary conditions at the cylinder walls. Equation (2.6f) is an additional kinematic boundary condition achieving the preservation of the interface. Finally, the formulation is closed by the Young-Laplace equation $(2.6 g)$ relating the pressure discontinuity at the interface to the capillary pressure.

This formulation represents a class of spectral sloshing problems well known in the literature. Faltinsen \& Timokha (2009) introduce different modal theories to find approximate analytical solutions for free-surface sloshing problems, mathematically quite similar to our two-layer formulation. In this study, we want to focus on first-order linear solutions, which are reasonable approximations as long as the wave amplitudes remain sufficiently small. In the linear approximation, the flow potentials $\phi_{i}$ can be described as superpositions of an infinite number of independent sloshing modes $m \in \mathbb{N}_{0}$ and $n \in \mathbb{N}_{1}$

$$
\begin{gathered}
\phi_{1}(r, \varphi, z, t)=-\sum_{m=0}^{\infty} \sum_{n=1}^{\infty} \Phi_{m n}(\varphi, t) \frac{\cosh \left(\frac{\epsilon_{m n}}{R}\left(z-h_{1}\right)\right)}{\sinh \left(\frac{\epsilon_{m n}}{R} h_{1}\right)} J_{m}\left(\epsilon_{m n} \frac{r}{R}\right), \\
\phi_{2}(r, \varphi, z, t)=\sum_{m=0}^{\infty} \sum_{n=1}^{\infty} \Phi_{m n}(\varphi, t) \frac{\cosh \left(\frac{\epsilon_{m n}}{R}\left(z+h_{2}\right)\right)}{\sinh \left(\frac{\epsilon_{m n}}{R} h_{2}\right)} J_{m}\left(\epsilon_{m n} \frac{r}{R}\right), \\
\text { with } \Phi_{m n}(\varphi, t)=\alpha_{m n}(t) \cos (m \varphi)+\beta_{m n}(t) \sin (m \varphi),
\end{gathered}
$$

which fulfil the Laplace equations and the no-outflow boundary conditions on the solid walls; $\alpha_{m n}(t)$ and $\beta_{m n}(t)$ are the time-dependent modal functions to be determined, $J_{m}$ are the $m$ th-order Bessel functions of the first kind and $\epsilon_{m n}$ denote the mode-dependent wavenumbers, restricted to the $n$ roots of the first derivative of the $m$ th-order Bessel function $\left(J_{m}^{\prime}\left(\epsilon_{m n}\right)=0\right.$; Abramowitz \& Stegun (1972)), needed to satisfy the no-outflow condition at the sidewalls; $\epsilon_{m n}$ are often called the radial wavenumbers since they determine the number of crests in the radial direction. Accordingly, $m$ denote the azimuthal wavenumbers.

In order for the flow potentials (2.7) to fulfil (2.6), we have to specify the modal functions $\alpha_{m n}(t)$ and $\beta_{m n}(t)$. Linear model theories show that the modal functions always obey a multidimensional system of ordinary differential equations. Faltinsen \& Timokha (2009, equation (5.155)) have derived the modal equations in the case of one fluid layer, which are mathematically very similar to our interfacial sloshing problem. By following their procedures, we find the modal equations

$$
\begin{aligned}
& \ddot{\alpha}_{1 n}(t)+2 \lambda_{1 n} \dot{\alpha}_{1 n}(t)+\omega_{1 n}^{2} \alpha_{1 n}(t)-\zeta_{n} \sin (\Omega t)=0 \\
& \ddot{\beta}_{1 n}(t)+2 \lambda_{1 n} \dot{\beta}_{1 n}(t)+\omega_{1 n}^{2} \beta_{1 n}(t)+\zeta_{n} \cos (\Omega t)=0
\end{aligned}
$$


with

$$
\begin{gathered}
\omega_{1 n}^{2}=\frac{\left(\rho_{2}-\rho_{1}\right) g \frac{\epsilon_{1 n}}{R}+\gamma\left(\frac{\epsilon_{1 n}}{R}\right)^{3}}{\rho_{1} \operatorname{coth}\left(\frac{\epsilon_{1 n}}{R} h_{1}\right)+\rho_{2} \operatorname{coth}\left(\frac{\epsilon_{1 n}}{R} h_{2}\right)}, \\
\zeta_{n}=\frac{\left(\rho_{2}-\rho_{1}\right) R d_{s} \Omega^{3}}{\left(\rho_{1} \operatorname{coth}\left(\frac{\epsilon_{1 n}}{R} h_{1}\right)+\rho_{2} \operatorname{coth}\left(\frac{\epsilon_{1 n}}{R} h_{2}\right)\right)\left(\epsilon_{1 n}^{2}-1\right) J_{1}\left(\epsilon_{1 n}\right)},
\end{gathered}
$$

to be fulfilled for $\alpha_{m n}(t)$ and $\beta_{m n}(t)$, where we have included linear damping rates $\lambda_{1 n}$ expressing the energy dissipation as explained by Faltinsen \& Timokha (2009, chap. 6). Here, $\omega_{1 n}^{2}$ are the natural eigenfrequencies of two-layer gravity-capillary waves in cylindrical tanks, while $\zeta_{n}$ can be understood as a mode-dependent forcing parameter describing the strength of excitation. Please note that only non-axisymmetric wave modes $(m=1)$ are excited in the linear regime, possessing exactly one nodal circle. Linear solutions do not exist for $m \neq 1$.

Equations (2.8a) and (2.8b) have the following stationary solutions:

$$
\begin{aligned}
& \alpha_{1 n}(t)=\frac{\zeta_{n}\left(\omega_{1 n}^{2}-\Omega^{2}\right)}{\left(\omega_{1 n}^{2}-\Omega^{2}\right)^{2}+4 \lambda_{1 n}^{2} \Omega^{2}} \sin (\Omega t)-\frac{2 \lambda_{1 n} \zeta_{n} \Omega}{\left(\omega_{1 n}^{2}-\Omega^{2}\right)^{2}+4 \lambda_{1 n}^{2} \Omega^{2}} \cos (\Omega t), \\
& \beta_{1 n}(t)=-\frac{\zeta_{n}\left(\omega_{1 n}^{2}-\Omega^{2}\right)}{\left(\omega_{1 n}^{2}-\Omega^{2}\right)^{2}+4 \lambda_{1 n}^{2} \Omega^{2}} \cos (\Omega t)-\frac{2 \lambda_{1 n} \zeta_{n} \Omega}{\left(\omega_{1 n}^{2}-\Omega^{2}\right)^{2}+4 \lambda_{1 n}^{2} \Omega^{2}} \sin (\Omega t) .
\end{aligned}
$$

Introducing these solutions $(2.11 a)$ and (2.11b) into (2.7a) and (2.7b) we can finally derive the flow potentials

$$
\begin{aligned}
& \phi_{1}(r, \varphi, z, t)=\sum_{n=1}^{\infty} \frac{\left(\rho_{2}-\rho_{1}\right) R d_{s} \Omega^{3}}{\left(\rho_{1} \operatorname{coth}\left(\frac{\epsilon_{1 n}}{R} h_{1}\right)+\rho_{2} \operatorname{coth}\left(\frac{\epsilon_{1 n}}{R} h_{2}\right)\right)} \frac{\cosh \left(\frac{\epsilon_{1 n}}{R}\left(z-h_{1}\right)\right)}{\sinh \left(\frac{\epsilon_{1 n}}{R} h_{1}\right)} \frac{J_{1}\left(\epsilon_{1 n} \frac{r}{R}\right)}{\left(\epsilon_{1 n}^{2}-1\right) J_{1}\left(\epsilon_{1 n}\right)} \\
& \times\left[\frac{\left(\omega_{1 n}^{2}-\Omega^{2}\right)}{\left(\omega_{1 n}^{2}-\Omega^{2}\right)^{2}+4 \lambda_{1 n}^{2} \Omega^{2}} \sin (\Omega t-\varphi)-\frac{2 \lambda_{1 n} \Omega}{\left(\omega_{1 n}^{2}-\Omega^{2}\right)^{2}+4 \lambda_{1 n}^{2} \Omega^{2}} \cos (\Omega t-\varphi)\right], \quad(2.12 a) \\
& \phi_{2}(r, \varphi, z, t)=\sum_{n=1}^{\infty} \frac{-\left(\rho_{2}-\rho_{1}\right) R d_{s} \Omega^{3}}{\left(\rho_{1} \operatorname{coth}\left(\frac{\epsilon_{1 n}}{R} h_{1}\right)+\rho_{2} \operatorname{coth}\left(\frac{\epsilon_{1 n}}{R} h_{2}\right)\right)} \frac{\cosh \left(\frac{\epsilon_{1 n}}{R}\left(z+h_{2}\right)\right)}{\sinh \left(\frac{\epsilon_{1 n}}{R} h_{2}\right)} \frac{J_{1}\left(\epsilon_{1 n} \frac{r}{R}\right)}{\left(\epsilon_{1 n}^{2}-1\right) J_{1}\left(\epsilon_{1 n}\right)} \\
& \times\left[\frac{\left(\omega_{1 n}^{2}-\Omega^{2}\right)}{\left(\omega_{1 n}^{2}-\Omega^{2}\right)^{2}+4 \lambda_{1 n}^{2} \Omega^{2}} \sin (\Omega t-\varphi)-\frac{2 \lambda_{1 n} \Omega}{\left(\omega_{1 n}^{2}-\Omega^{2}\right)^{2}+4 \lambda_{1 n}^{2} \Omega^{2}} \cos (\Omega t-\varphi)\right] . \quad(2.12 b)
\end{aligned}
$$

The velocity fields in the moving reference frame of the cylinder can be calculated using (2.5). The interface elevation $\eta$ is determined by linearising (2.6f). It can be expressed as

$$
\begin{aligned}
& \eta(r, \varphi, t)=\sum_{n=1}^{\infty} \frac{\left(\rho_{2}-\rho_{1}\right) d_{s} R \Omega^{2}}{\left[\left(\rho_{2}-\rho_{1}\right) g+\left(\frac{\epsilon_{1 n}}{R}\right)^{2} \gamma\right]} \frac{J_{1}\left(\epsilon_{1 n} \frac{r}{R}\right)}{\left(\epsilon_{1 n}^{2}-1\right) J_{1}\left(\epsilon_{1 n}\right)} \\
& \quad \times\left[\frac{\omega_{1 n}^{2}\left(\omega_{1 n}^{2}-\Omega^{2}\right)}{\left(\omega_{1 n}^{2}-\Omega^{2}\right)^{2}+4 \lambda_{1 n}^{2} \Omega^{2}} \cos (\Omega t-\varphi)+\frac{2 \lambda_{1 n} \Omega \omega_{1 n}^{2}}{\left(\omega_{1 n}^{2}-\Omega^{2}\right)^{2}+4 \lambda_{1 n}^{2} \Omega^{2}} \sin (\Omega t-\varphi)\right] .
\end{aligned}
$$


As a main difference from the previous inviscid theories, the azimuthal part of our solution is composed of both a symmetric $\sim \cos (\Omega t-\varphi)$ and an antisymmetric $\sim \sin (\Omega t-\varphi)$ contribution, whereas inviscid solutions are always symmetric. In the limit $\rho_{2}, \lambda_{1 n}, \gamma \mapsto 0$ this solution is equivalent to the inviscid one-layer theory by Reclari et al. (2014), as we prove in appendix B. From (2.13) we can deduce the resonance frequencies $\omega_{R, n}$ of the wave modes which are modified by damping. Close to resonance $\left(\Omega \approx \omega_{1 n}\right)$ the interface elevation is described only by the antisymmetric part of the solution. Therefore

$$
\left.\eta(r, \varphi=\Omega t-\pi / 2, t)\right|_{\Omega \approx \omega_{1 n}}=A \frac{2 \lambda_{1 n} \Omega^{3} \omega_{1 n}^{2}}{\left(\omega_{1 n}^{2}-\Omega^{2}\right)^{2}+4 \lambda_{1 n}^{2} \Omega^{2}},
$$

where $A$ contains the frequency-independent prefactors of (2.13). The shaking frequency causing the highest amplitude $\left(\Omega \equiv \omega_{R, n}\right)$ is calculated by solving

$$
\frac{\partial \eta(r, \varphi=\Omega t-\pi / 2, t)}{\partial \Omega}=0
$$

giving a resonance frequency of

$$
\omega_{R, n}^{2}=2 \sqrt{\left(\omega_{1 n}^{2}-\lambda_{1 n}^{2}\right)^{2}+\lambda_{1 n}^{2} \omega_{1 n}^{2}}-\omega_{1 n}^{2}+2 \lambda_{1 n}^{2} \geqslant \omega_{1 n}^{2} .
$$

Thus, high damping rates always increase the resonance frequency. This behaviour might appear counterintuitive and is contrary to that of the classical driven harmonic oscillator. It is caused by differences of the external forcing. The forcing amplitude itself depends on the driving frequency and scales with $\sim \Omega^{3}$ (see (2.10)), introducing the tendency that the wave is generally amplified for higher shaking frequencies independently of the resonant amplifications. This effect overcompensates the common frequency drop observed in systems underlying a constant driving force ( $\zeta_{n}$ independent of $\Omega$ ). However, in practical applications, as with OSBs, a noticeable increase of the eigenfrequency will not be observable for the first modes due to their weak dependency on $\lambda_{1 n}$ in (2.16) for common damping rates $\lambda_{1 n} \lesssim 1$. An increase of $\omega_{R, n}$ would be observable only in highly overdamped set-ups. For weakly damped systems we identify $\omega_{R, n} \approx \omega_{1 n}$ (at least for the first important large-scale wave modes).

\subsection{Viscous damping}

Equation (2.13) is the final result of the modal analysis containing the responding resonance dynamics of the interface. In contrast to previous inviscid theories (Reclari et al. 2014; Bouvard et al. 2017), our solution does not contain any singularities at the resonance frequencies. Those are resolved by the damping parameters $\lambda_{1 n}$ determining the maximum amplitudes at resonance $\Omega \approx \omega_{1 n}$. However, using an irrotational approach, the damping rates cannot be further deduced since viscous dissipation is caused by the rotational part of the flow manifested in the boundary layers. Therefore, damping rates must be determined a priori, i.e. by fitting the exponential decay of resonant waves after the shaker is switched off. Then, equation (2.13) can predict the wave amplitudes (within its limits) for all shaking frequencies $\Omega$ around the considered resonance frequency.

However, to allow for further analysis and a better understanding of how viscous damping can affect the resonance dynamics, it is desirable to describe the wave 
elevation in direct dependence of the viscosities $v_{i}$. For that purpose, we exploit some of the recent results given by Herreman et al. (2019), who applied a perturbation approach to study interfacial wave damping. They derived viscous damping rates of free gravity-capillary waves by explicitly calculating Stokes boundary layers for the same two-layer geometry that we are considering in the present paper. It was found in this study that viscous damping rates of free-surface and interfacial waves are inherently different, so that we must carefully distinguish between the one- and two-layer limit of viscous damping rates in the following.

In the first order, the two-layer damping rate $\lambda_{1 n}^{2 L}$ is composed of three different contributions

$$
\lambda_{1 n}^{2 L}=\lambda_{1 n}^{B L}+\lambda_{1 n}^{I L}+\lambda_{1 n}^{I n t},
$$

with

$$
\begin{aligned}
& \lambda_{1 n}^{B L}=\sum_{i=1,2} \rho_{i} \sqrt{\frac{\nu_{i} \Omega}{8 R^{2}}}\left[\frac{\epsilon_{1 n}\left(1-\frac{h_{i}}{R}\right) \sinh ^{-2}\left(\frac{\epsilon_{1 n}}{R} h_{i}\right)+\left(\frac{\epsilon_{1 n}^{2}+1}{\epsilon_{1 n}^{2}-1}\right) \operatorname{coth}\left(\frac{\epsilon_{1 n}}{R} h_{i}\right)}{\rho_{1} \operatorname{coth}\left(\frac{\epsilon_{1 n}}{R} h_{1}\right)+\rho_{2} \operatorname{coth}\left(\frac{\epsilon_{1 n}}{R} h_{2}\right)}\right], \\
& \lambda_{1 n}^{I L}=\frac{\epsilon_{1 n}}{\left(\frac{1}{\rho_{1}} \sqrt{\frac{8 R^{2}}{\Omega v_{1}}}+\frac{1}{\rho_{2}} \sqrt{\frac{8 R^{2}}{\Omega v_{2}}}\right)}\left[\frac{\left(\operatorname{coth}\left(\frac{\epsilon_{1 n}}{R} h_{1}\right)+\operatorname{coth}\left(\frac{\epsilon_{1 n}}{R} h_{2}\right)\right)^{2}}{\rho_{1} \operatorname{coth}\left(\frac{\epsilon_{1 n}}{R} h_{1}\right)+\rho_{2} \operatorname{coth}\left(\frac{\epsilon_{1 n}}{R} h_{2}\right)}\right], \\
& \lambda_{1 n}^{I n t}=\frac{2 \epsilon_{1 n}^{2}\left(\frac{\rho_{2} v_{2}}{R^{2}}-\frac{\rho_{1} v_{1}}{R^{2}}\right)}{\left(\frac{1}{\rho_{1} \sqrt{\nu_{1}}}+\frac{1}{\rho_{2} \sqrt{\nu_{2}}}\right)}\left[\frac{\frac{1}{\rho_{1} \sqrt{\nu_{1}}} \operatorname{coth}\left(\frac{\epsilon_{1 n}}{R} h_{2}\right)-\frac{1}{\rho_{2} \sqrt{\nu_{2}}} \operatorname{coth}\left(\frac{\epsilon_{1 n}}{R} h_{1}\right)}{\left(\rho_{1} \operatorname{coth}\left(\frac{\epsilon_{1 n}}{R} h_{1}\right)+\rho_{2} \operatorname{coth}\left(\frac{\epsilon_{1 n}}{R} h_{2}\right)\right)}\right] .
\end{aligned}
$$

The first contribution $\lambda_{1 n}^{B L}$ involves viscous dissipation arising at the solid tank boundary layers including the side, top and bottom walls. The second term $\lambda_{1 n}^{I L}$ describes the dissipation rate in the interfacial boundary layers above and below the interface. Finally, $\lambda_{1 n}^{I n t}$ is the interior damping rate which can be destabilising. Please note that this damping rate is fundamentally different from the well-known damping rates of free-surface waves in upright cylinders calculated by Case \& Parkinson (1956) and Miles \& Henderson (1998). The first solid boundary layer term $\lambda_{1 n}^{B L}$ is physically the same as for one-layer systems. The one-layer limit $\rho_{1} \mapsto 0$ of $\lambda_{1 n}^{B L}$ is equivalent to the boundary layer term derived by Case \& Parkinson (1956). However, the interfacial layer contribution $\lambda_{1 n}^{I L}$ does not exist in free-surface waves in the leading order. The reason is that the flow field under free surfaces is to a good approximation irrotational, which is not the case around moving interfaces, where viscous boundary layers evolve to balance the strong tangential shear flows between the liquids. Indeed, we find $\lambda_{1 n}^{I L} \mapsto 0$ for $\rho_{1} \mapsto 0$. For liquids with similar densities and viscosities $\lambda_{1 n}^{B L}$ and $\lambda_{1 n}^{I L}$ can have comparable magnitudes and should always both be considered. In contrast, the interior damping rate $\lambda_{1 n}^{I n t}$ is completely negligible for liquids with densities of the same order. However, this term cannot be ignored anymore when we approach the free-surface limit $\rho_{1} \mapsto 0$. Then, the interior damping is increased by orders of magnitude until it reaches the limit $\lambda_{1 n}^{\operatorname{Int}} \mapsto 2 \nu_{2} \epsilon_{1 n}^{2} / R^{2}$, the familiar interior damping rate of free-surface waves that is generally not negligible. This is the only remaining dissipation term for irrotational surface waves, while 
interfacial wave damping is always manifested in rotational boundary layers. All in all, leading-order interfacial damping is well described by

$$
\lambda_{1 n}^{2 L}=\lambda_{1 n}^{B L}+\lambda_{1 n}^{I L}
$$

In contrast, free-surface damping must be calculated using the contributions

$$
\lambda_{1 n}^{1 L}=\lambda_{1 n}^{B L}+\tilde{\lambda}_{1 n}^{I n t}
$$

with

$$
\begin{gathered}
\lambda_{1 n}^{B L}=\sqrt{\frac{v_{2} \Omega}{8 R^{2}}}\left[\frac{\epsilon_{1 n}^{2}+1}{\epsilon_{1 n}^{2}-1}+\frac{2 \epsilon_{1 n}\left(1-\frac{h_{2}}{R}\right)}{\sinh \left(2 \frac{\epsilon_{1 n}}{R} h_{2}\right)}\right], \\
\tilde{\lambda}_{1 n}^{I n t}=\frac{v_{2}}{R^{2}}\left[2 \epsilon_{1 n}^{2}-\frac{1}{\epsilon_{1 n}^{2}-1}\left(1+\frac{2 \epsilon_{1 n} h_{2}}{R \sinh \left(2 \frac{\epsilon_{1 n}}{R} h_{2}\right)}\right)\right] .
\end{gathered}
$$

The boundary layer damping (2.23) is the one-layer limit of (2.18), whereas the interior damping rate $\tilde{\lambda}_{1 n}^{\text {Int }}$ was supplemented by an additional sidewall contribution calculated by Miles \& Henderson (1998) in order to describe damping with the best known accuracy. This contribution is entirely negligible for interfacial waves and still small for typical free-surface cylinders such as OSBs. In this form, the damping rate (2.22) is fully equivalent to the formulation recently used by Raynovskyy \& Timokha (2018a) to study resonant sloshing. In the shallow water limit, the damping rate is further consistent with the Stokes wall shear stress model presented by Alpresa et al. $(2018 b)$, predicting a spiral-like shape of the horizontal velocity profile in the bottom boundary layer.

Technically, both damping rates $\lambda_{1 n}^{2 L}$ and $\lambda_{1 n}^{1 L}$ are only valid for free gravity-capillary waves; dissipation rates of forced wave motion are generally more complex. However, damping affects linear waves mainly in a small window around the eigenfrequencies $\Omega \approx \omega_{1 n}$, as we will show in $\S 2.4$. In this frequency range, the interfacial motions are very similar to free wave motions and dissipation rates can be well approximated by (2.17) and (2.22). This is not generally the case for frequencies far below or above the resonance frequency, but there, damping is of no importance except for a largely overdamped system.

Finally, it is important to note that the damping rates do not involve any dissipation mechanism associated with the contact line (contact line hysteresis, meniscus dynamics) or surface contamination. Hence, (2.17) and (2.22) are always expected to slightly underestimate viscous damping rates and should be considered as conservative estimations to predict maximum expectable interface elevations. While it is often difficult to realise two-layer stratifications involving free-sliding contact lines and negligible menisci (Horstmann et al. 2019), these idealised boundary conditions apply for most mid- and large-size free-surface systems, including typical commercial OSBs.

\subsection{Dimensionless prediction of resonance dynamics}

In this section we want to relate the wave solutions (2.12b) and (2.13) to the eight dimensionless parameters introduced in $\$ 2.1$ in order to discuss the damped wave 
dynamics predicted by our model. We can write the solutions in dimensionless forms by introducing the dimensionless variables $\tilde{r}=r / R, \tilde{z}=z / R$ and $\tilde{t}=\Omega t$. This way, the interface elevation $\eta(2.13)$ can be expressed as

$$
\begin{aligned}
& \frac{\eta(\tilde{r}, \varphi, \tilde{t})}{R}=\sum_{n=1}^{\infty} \frac{2 F r}{\left(1+\frac{\epsilon_{1 n}^{2}}{B o}\right)} \frac{J_{1}\left(\epsilon_{1 n} \tilde{r}\right)}{\left(\epsilon_{1 n}^{2}-1\right) J_{1}\left(\epsilon_{1 n}\right)} \\
& \quad \times\left[\frac{\Gamma_{\omega_{1 n}}^{2}}{\frac{\left(\Gamma_{\omega_{1 n}}^{2}-1\right)^{2}}{\Lambda_{1 n}}+\Lambda_{1 n}} \sin (\tilde{t}-\varphi)+\frac{\Gamma_{\omega_{1 n}}^{2}}{\left(\Gamma_{\omega_{1 n}}^{2}-1\right)+\frac{\Lambda_{1 n}^{2}}{\left(\Gamma_{\omega_{1 n}}^{2}-1\right)} \cos (\tilde{t}-\varphi)}\right],
\end{aligned}
$$

where $\Gamma_{\omega_{1 n}}\left(F r, E, B o, H_{i}, A\right)=\omega_{1 n} / \Omega$ and $\Lambda_{1 n}\left(R e_{i}, H_{i}, A\right)=2 \lambda_{1 n} / \Omega$. Full formulas for these dimensionless frequencies and damping rates as well as for the dimensionless potentials and velocities are given in appendix A. Equation (2.25) is the final result of our theoretical work, allowing analysis of the wave elevation as a function of all eight dimensionless input parameters. The physics contained within this solution is very rich, although it may be difficult to recognise in the presented form. By analysing the different wave contributions and the asymptotics, we can further deduce various wave properties. One main difference between this damped wave solution in comparison with the previous inviscid description (Reclari et al. 2014) is the existence of an antisymmetric part $\sim \sin (\tilde{t}-\varphi)$, not present in the inviscid solution. The superposition of the symmetric and antisymmetric solutions captures the phase shift $\Delta \varphi$ between the shaking table and the responding wave. The phase $\varphi^{*}$ of the maximum wave elevation can be calculated by solving

$$
\frac{\partial \eta\left(\tilde{r}, \varphi^{*}, \tilde{t}\right)}{R \partial \varphi^{*}}=0 .
$$

From this, we find a phase difference of

$$
\Delta \varphi(\tilde{r})=\arctan \left(\frac{\sum_{n=1}^{\infty} \frac{1}{\left(B o+\epsilon_{1 n}^{2}\right)} \frac{J_{1}\left(\epsilon_{1 n} \tilde{r}\right)}{\left(\epsilon_{1 n}^{2}-1\right) J_{1}\left(\epsilon_{1 n}\right)} \frac{\Lambda_{1 n} \Gamma_{\omega_{1 n}}^{2}}{\left(\Gamma_{\omega_{1 n}}^{2}-1\right)^{2}+\Lambda_{1 n}^{2}}}{\sum_{n=1}^{\infty} \frac{1}{\left(B o+\epsilon_{1 n}^{2}\right)} \frac{J_{1}\left(\epsilon_{1 n} \tilde{r}\right)}{\left(\epsilon_{1 n}^{2}-1\right) J_{1}\left(\epsilon_{1 n}\right)} \frac{\Gamma_{\omega_{1 n}}^{2}\left(\Gamma_{\omega_{1 n}}^{2}-1\right)}{\left(\Gamma_{\omega_{1 n}}^{2}-1\right)^{2}+\Lambda_{1 n}^{2}}}\right) .
$$

The phase difference depends on the radial coordinate $\tilde{r}$ since the crests of the individual nodal cycles $n$, which are located at different radial positions, can be shifted differently by the mode-dependent damping rates (2.17). Considering only the phase shift of the first mode $(n=1)$, equation (2.27) simplifies to

$$
\left.\Delta \varphi\right|_{n=1}=\arctan \left(\frac{\Lambda_{11}}{\Gamma_{\omega_{11}}^{2}-1}\right)=\arctan \left(\frac{2 \lambda_{11} \Omega}{\omega_{11}^{2}-\Omega^{2}}\right),
$$

exactly reproducing the well-known phase shift of the driven harmonic oscillator, even though we found a different resonance frequency (see (2.16)). Equation (2.28) can be exploited to estimate the linear out-of-phase flow. By defining an expedient critical 
small phase shift $\Delta \varphi>\Delta \varphi_{c}$ as an out-of-phase threshold, a corresponding critical shaking frequency $\Omega_{c}$ for out-of-phase flow can be calculated as

$$
\frac{\Omega_{c}}{\omega_{11}} \geqslant \sqrt{\frac{1}{\Lambda_{11} \cot \left(\Delta \varphi_{c}\right)+1}} .
$$

It becomes apparent that the linear phase shift is primarily specified by the damping forces.

Likewise, the maximum achievable interface elevation for given system parameters can be estimated from (2.25). Close to resonance the wave elevation is determined only by the antisymmetric part of the solution (2.25). Here, we can locate the maximum elevation $\eta_{0} / R$ of the first dominant mode at $\tilde{r}=1, \varphi=\tilde{t}-\pi / 2$ and $\Omega=\omega_{11}$, yielding

$$
\frac{\eta_{0}}{R}=\frac{2 F r}{\left(1+\frac{\epsilon_{1 n}^{2}}{B o}\right)} \frac{1}{\epsilon_{11}^{2}-1} \frac{\Gamma_{\omega_{11}}^{2}}{\Lambda_{11}}
$$

This expression is only valid for finite amplitudes. Nonlinear effects and wave breaking cause additional dissipation, effectively reducing the maximal elevation, an effect not captured by the theory. However, equation (2.30) is a good measure for the maximum attainable wave elevation. As pointed out by Reclari (2013) and Alpresa et al. (2018a), linear potential theory can adequately predict free-surface motions up to a critical amplitude, where breaking is expected. This usually happens if the wave steepness exceeds a critical value beyond which gravity waves become unstable. For the first and practically most important wave mode $\epsilon_{11}$, the critical wave steepness $\eta_{0} / R \gtrsim 0.44$ has been well established as a wave breaking criterion in orbitally shaken cylinders with deep fluid layers (Reclari 2013). Up to this wave slope, equation (2.30) is expected to predict the resonant interfacial displacement with sufficient accuracy. However, this criterion is not valid for shallow layers, where the vicinity of the bottom leads to additional effects including a partially uncovered bottom. For these cases Alpresa et al. (2018a) verified the shallow breaking criterion $\eta_{0} / R \gtrsim 0.7 H$. By combining these criteria as upper thresholds and by modifying (2.30) according to (A4) and (A 5), we can derive the following relations for the resonant wave slope within the one-layer limit as relevant for OSBs:

$$
\begin{aligned}
& \frac{\eta_{0}}{R}=\chi \sqrt{\operatorname{Re} E} \text { if } \begin{cases}\frac{\eta_{0}}{R}<0.44 & \text { for } H \gtrsim 0.63 \\
\frac{\eta_{0}}{R}<0.7 H & \text { for } H \lesssim 0.63\end{cases}
\end{aligned}
$$

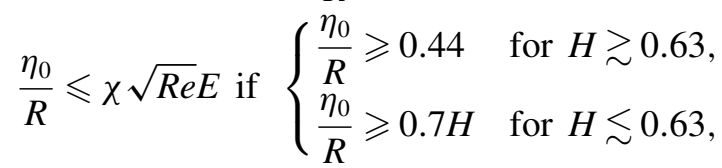

with the form factors

$$
\chi=\frac{\sqrt{8} \epsilon_{11} \sinh ^{2}\left(\epsilon_{11} H\right)}{\epsilon_{11}\left(\epsilon_{11}^{2}-1\right)(1-H)+\sinh \left(\epsilon_{11} H\right) \cosh \left(\epsilon_{11} H\right)\left(\epsilon_{11}^{2}+1\right)} .
$$

Here, the index $i=2$ was omitted for better readability. In line with a conservative estimation, the interior damping distribution $\sim R e$ in (2.22) was ignored since it is, in 
comparison with boundary layer damping, negligibly small for lower wave modes in OSB-size cylinders $(R \sim 10 \mathrm{~cm})$. The maximum wave displacement is independent of the gravity force and surface tension. It is solely determined by the balance of driving and viscous forces. We can determine the relation $\sqrt{R e} E=d_{s} /(2 \delta)$, showing that the maximum slope scales approximately with the length ratio between the shaking radius $d_{s} / 2$ and the typical boundary layer thickness $\delta$. This scaling is precisely valid for interfacial waves, where interior damping is always negligible. The implied scaling law $\eta_{0} / R \sim R e^{\kappa}$ with $\kappa=0.5$ can generally be considered as an upper threshold also for non-resonant excitations since, at resonance, the impact of damping forces is always at a maximum. We finally conclude that an upper value of the scaling coefficient of $\kappa \leqslant 0.5$ can be expected for most OSBs and all two-layer systems depending on the shaking frequency $\Omega$ and on nonlinearities.

Lastly, we want to analyse the wave character of the elevated free surface. For low shaking frequencies $\Omega$ sufficiently below the first eigenfrequency $\omega_{11}$ the displaced free surface has the form of a tilted disk rather than a wavy shape (Reclari 2013; Weheliye et al. 2013; Ducci \& Weheliye 2014). The transition from the flat disk shape to the waveform is important for OSBs since the mixing dynamics is changed considerably: as soon as the surface becomes wavy, the nonlinear out-of-phase flow regime starts to develop (Weheliye et al. 2013).

As shown in appendix B, the symmetric part of solution (2.25) can be transformed into the following form:

$$
\frac{\eta(\tilde{r}, \varphi, \tilde{t})}{R}=2 F r\left[\frac{\tilde{r}}{2}+\sum_{n=1}^{\infty} \frac{\left(\Gamma_{\omega_{1 n}}^{2}-1\right)-\Lambda_{1 n}^{2}}{\left(\Gamma_{\omega_{1 n}}^{2}-1\right)^{2}+\Lambda_{1 n}^{2}} \frac{J_{1}\left(\epsilon_{1 n} \tilde{r}\right)}{\left(\epsilon_{1 n}^{2}-1\right) J_{1}\left(\epsilon_{1 n}\right)}\right] \cos (\tilde{t}-\varphi),
$$

when surface tension is neglected $(B o \gg 1)$. The radial part of (2.32) is composed of a linear contribution $\sim \tilde{r}$ representing the disk and a wavy contribution $\sim J_{1}\left(\epsilon_{1 n} \tilde{r}\right)$ resulting from the superposition of free gravity wave modes. We can now compare the linear contribution with the wave-like contributions. We consider a fixed time point $\tilde{t}=0$ and the radial position $\tilde{r}=1$, where both the disc and the first wave mode are of maximum elevation. Further restricting ourselves to small shaking frequencies $\Omega$ below the first resonance frequency $\omega_{11}$ allows us to keep only the first wave mode $(n=1)$. We can then compare the wavy parts (mode $n=1$ in (2.32) and the antisymmetric part of (2.25)) with the disc component and calculate the point where the sum of the wavy components matches a certain percentage $\Upsilon$ of the disc solution

$$
\frac{\Upsilon}{2}=\frac{\left(\Gamma_{\omega_{11}}^{2}-1\right)-\Lambda_{11}^{2}}{\left(\Gamma_{\omega_{11}}^{2}-1\right)^{2}+\Lambda_{11}^{2}} \frac{1}{\left(\epsilon_{11}^{2}-1\right)}-\frac{\Lambda_{11} \Gamma_{\omega_{11}}^{2}}{\left(\Gamma_{\omega_{11}}^{2}-1\right)^{2}+\Lambda_{11}^{2}} \frac{1}{\left(\epsilon_{11}^{2}-1\right)} \tan \left(\varphi^{*}\right) .
$$

The phase $\varphi^{*}$ of maximum wave elevation can be calculated in analogy to (2.27) and is given by

$$
\varphi^{*}=\arctan \left(-\frac{\Lambda_{11}}{\Gamma_{\omega_{11}}^{2}-1}\right) .
$$

By inserting (2.34) into (2.33) the wave percentage simplifies to

$$
\Upsilon=\frac{2}{\left(\epsilon_{11}^{2}-1\right)} \frac{1}{\left(\Gamma_{\omega_{11}}^{2}-1\right)}
$$

The damping parameter $\Lambda_{11}$ does not occur in (2.35) and thus the wave fraction $\Upsilon$ is independent of the system's damping. This somewhat surprising result implies that the 
disc and the first wave mode are equally damped. Hence, damping will not influence the plane-wave transition. Equation (2.35) can be derived as well by directly using the inviscid solution (equation (2.32) with $\Lambda_{11}=0$ ). Any possible influence of viscosity to the transition would be manifested in the rotational part of the flow confined to the boundary layers.

By including the dimensionless frequency (A 4) in (2.35) we can derive the critical Froude number $F r_{\text {wave }}$ needed to induce a certain waviness $\Upsilon$

$$
F r_{\text {wave }}(\Upsilon)=\frac{E \epsilon_{11} \tanh \left(\epsilon_{11} H\right)}{2\left(\epsilon_{11}^{2}-1\right)^{-1} \Upsilon^{-1}+1} .
$$

This formula can be used to predict the limits of the linear scaling law $\eta_{0} / R \sim F r$ proposed by Weheliye et al. (2013) and Ducci \& Weheliye (2014) as well as the nonlinear out-of-phase flow regime in OSBs, as we will discuss in $\S 4.3$.

\subsection{Limits of applicability}

Two of the initial assumptions are mainly responsible for the limits of our theoretical model: irrotationality and linearity. Even though the dissipative effect of rotational boundary layers is incorporated in our model by means of the damping rate formula (2.17), we neglect the influences of the boundary layers on the wave shapes and the velocity fields in the bulk since the flow solutions violate the no-slip boundary conditions. Hence, the wave modes (2.25) can describe the bulk flow only adequately if the boundary layer thicknesses $\delta_{i}$ are far smaller than the internal dimensions

$$
\delta_{i} \sim \sqrt{\frac{\nu_{i}}{\Omega}}=\frac{R}{\sqrt{R e_{i}}} \ll R .
$$

This estimate directly leads to the condition

$$
\sqrt{R e_{i}} \gg 1
$$

which is no serious limitation in practice. Even small-scale bioreactors rarely operate at $R e \lesssim 10^{2}$ (Alpresa et al. 2018a) since the fluid-driving wave would otherwise be too small to generate sufficient mixing.

A more serious restriction is the linearisation limiting our model to fairly small amplitude waves. Nonlinear effects such as subharmonic wave modes (Reclari et al. 2014), the mean flow (Bouvard et al. 2017) and turbulent wave dynamics are not captured. As discussed before, the wave breaking conditions

$$
\frac{\eta_{0}}{R} \lesssim \begin{cases}0.44, & \text { for } \min \left(H_{1}, H_{2}\right) \gtrsim 0.63, \\ 0.7 \min \left(H_{1}, H_{2}\right), & \text { for } \min \left(H_{1}, H_{2}\right) \lesssim 0.63,\end{cases}
$$

are good estimates for linearity since good agreement with the inviscid potential model was found for the single-crested $n=1$ mode in this amplitude regime. For OSBs we can use (2.31) to derive an upper threshold for the Reynolds number

$$
\sqrt{\operatorname{Re}} \lesssim \begin{cases}\frac{0.44}{\chi E}, & \text { for } H \gtrsim 0.63 \\ \frac{0.7 H}{\chi E}, & \text { for } H \lesssim 0.63\end{cases}
$$


Consequently, a critical eccentricity $E$ can be estimated beyond which nonlinear waves might evolve for excitation frequencies lower than or equal to the resonance frequency. In practice, condition (2.40) is fulfilled for small-scale OSBs of order $R \sim 1 \mathrm{~cm}$ as used for protein degradation. But it is violated for large-scale bioreactors of orders $R \sim 10$ up to $R \sim 100 \mathrm{~cm}$, as commonly used, e.g. for mammalian cell cultivation (Klöckner et al. 2012, 2014). However, equation (2.40) is a conservative limit for the maximum attainable amplitudes at resonance. For excitation frequencies sufficiently below or above the resonance frequency, amplitudes are considerably lower and our theory can predict wave amplitudes up to a critical Froude number, as previously shown for the inviscid model (Reclari et al. 2014). Some of the results, such as the prediction of the waviness (2.36), are of practical relevance for large-scale OSBs as well; see $\S 4.3$ for details.

An inequality similar to (2.39) applies for the two-layer case, albeit with different wave breaking limits, which can depend on the density difference. Even so, an application to two-layer systems extends the valid parameter range of our model in comparison with equivalent one-layer waves (Horstmann et al. 2019). Here, the model performs best. This is mainly due to the density differences, which are considerably lower for most liquid-liquid interfaces compared with gas-liquid free-surfaces. Typical oil-water combinations possess up to an order of magnitude lower eigenfrequencies compared with free surfaces. In consequence, the wave amplitudes for frequencies near the resonance frequency, where the elevation scales with $\sim \Omega \omega_{1 n}^{2}$ (cf. (2.13)), are much smaller.

Concluding this section, we have to emphasise again that our theory cannot describe contact line dynamics. The theory is valid only for idealised boundary conditions (free-sliding contact line with a static contact angle of $90^{\circ}$ ). This condition is in good approximation fulfilled for many mid- and large-scale surface-wave systems but harder to realise for interfacial waves (cf. Horstmann et al. 2019). For the latter case, contact line hysteresis and dynamic menisci can frequently be observed and pose great challenges to modelling. For the idealised case of a perfectly fixed contact line there are some wave theories in the literature (Miles 1991; Henderson \& Miles 1994). Very recently, Viola \& Gallaire (2018) succeeded in developing a mathematical framework which incorporates a dynamic contact angle model. Formulating these approaches under orbital excitation is a promising task that we have to leave for future studies.

\section{Numerical results}

The theory introduced in the preceding paragraphs contains a rich wave dynamics which we present in this section using illustrative examples. Wave shapes depending on mode number and damping rate are discussed first and then related to forcing frequency and radial position. The section concludes with an overview of the influence of the dimensionless parameters on the wave amplitudes around the first resonance frequency.

We use the paraffin oil-silicon oil experiment described in Horstmann et al. (2019) as a default case around which we modify different parameters to study the damped wave dynamics. A cylinder of equal height and diameter of $10 \mathrm{~cm}$ was used and excited with shaking frequencies ranging from 20 up to 90 revolutions per minute (r.p.m.) with a default shaking diameter of $d_{s}=2.5 \mathrm{~cm}$. All relevant physical parameters and corresponding dimensionless numbers are listed in table 1.

First, we analyse how damping forces influence the shapes of different wave modes. The interfacial elevation is given by (2.13) and changes with the driving frequency $\Omega$. 


$\begin{array}{lcccccc}\text { Property } & d_{s}(\mathrm{~cm}) & \rho_{i}\left(\mathrm{~g} \mathrm{~cm}^{-3}\right) & \gamma\left(\mathrm{mN} \mathrm{m}^{-1}\right) & v_{i}\left(\mathrm{~m}^{2} \mathrm{~s}^{-1}\right) & h_{i}(\mathrm{~cm}) & R(\mathrm{~cm}) \\ \text { Layer 1 } & 2.5 & 846 & \approx 20 & \approx 36 & 5 & 5 \\ \text { Layer 2 } & 2.5 & 955 & \approx 20 & \approx 35 & 5 & 5 \\ \text { Number } & F r & E & R e_{i} & H_{i} & B o & A \\ \text { Layer 1 } & 0.027 & 0.25 & 320 & 1 & 134 & 0.06 \\ \text { Layer 2 } & 0.027 & 0.25 & 330 & 1 & 134 & 0.06\end{array}$

TABLE 1. Default parameters and physical properties of the Paraffinum Perliquidum |Wacker ${ }^{\circledR}$ silicone oil AK 35 interfacial wave experiment described in Horstmann et al. (2019). The resulting dimensionless numbers, calculated for the first eigenfrequency $\Omega=$ $\omega_{11} \approx 44$ r.p.m., are shown in addition.

This solution is composed of a linear contribution (flat disc) and the $n$ wave solutions reflected by the wavenumbers $\epsilon_{1 n}$, which are most prominent at the eigenfrequencies $\omega_{1 n}$. Figure 2 shows the normalised interface elevations calculated at the first four eigenfrequencies $\Omega=\left(\omega_{11}, \omega_{12}, \omega_{13}, \omega_{14}\right)$ for four different damping parameters $\lambda_{1 n}=$ $(0,0.5,1,2.5) \mathrm{s}^{-1}$. The left column displays the inviscid solutions of the first four modes already presented in Reclari (2013). It can be seen how the number of crests and troughs increases with the radial wavenumber and the maximum wave elevations become more and more concentrated in the centre of the cylinder.

The three other columns display wave modes under the influence of damping forces. As visible in the second $\left(\lambda_{1 n}=0.5 \mathrm{~s}^{-1}\right)$ and third $\left(\lambda_{1 n}=1 \mathrm{~s}^{-1}\right)$ columns, damping causes a twisting of the $n$ nodal circles such that spiral wave structures evolve, clearly visible in the higher modes $n \geqslant 2$. The effect of damping is weak for the first mode $n=1$, where the crest-trough symmetry is almost kept intact. But for higher modes the initially separated crests and troughs begin to merge under the influence of damping until only one clearly distinguishable crest-trough pair survives for $\lambda_{1 n}=1 \mathrm{~s}^{-1}$, appearing as a coherent spiral. With increasing damping, the maximum elevations move towards the cylinder wall until the wave is completely damped out in the cell centre for $\lambda_{1 n}=2.5 \mathrm{~s}^{-1}(n=3,4)$. For $\lambda_{1 n}=2.5 \mathrm{~s}^{-1}$ the wave is already completely overdamped and condition (2.38) might no longer be fulfilled. In such cases, the boundary layers begin to grow into the bulk and would suppress interfacial displacements near the sidewalls, making it unlikely that these waveforms can be observed in experiments.

The transition to a spiral wave can also be understood mathematically. It is due to the mode-dependent phase lags which are governed by (2.27). This equation predicts that all nodal cycles underlie individual phase lags with regard to the shaking table, causing phase shifts between the single modes. These individual phase shifts result in mutual twists of the $n$ nodal cycles, finally forming the coherent spirals.

Now, we want to graphically study the resonance dynamics covering the predicted maximum attainable wave amplitudes $\eta_{0}$ in dependence of the shaking frequencies $\Omega$. At first, we compare the interfacial elevations of the first two wave modes at different interfacial locations. Figure 3 shows the maximum dimensionless interface elevation $\eta_{0} / R$ in dependence of the dimensionless shaking frequency $\Omega / \omega_{11}$ at two different radial positions: close to the cell centre $\tilde{r}=r / R=0.1$ (coloured in blue) and at the sidewall $\tilde{r}=1$ (coloured in magenta). In addition, the dashed black curves show the corresponding inviscid solution for both positions. As expected, the inclusion of damping forces has closed the resonance curves. Non-physical singularities at the 


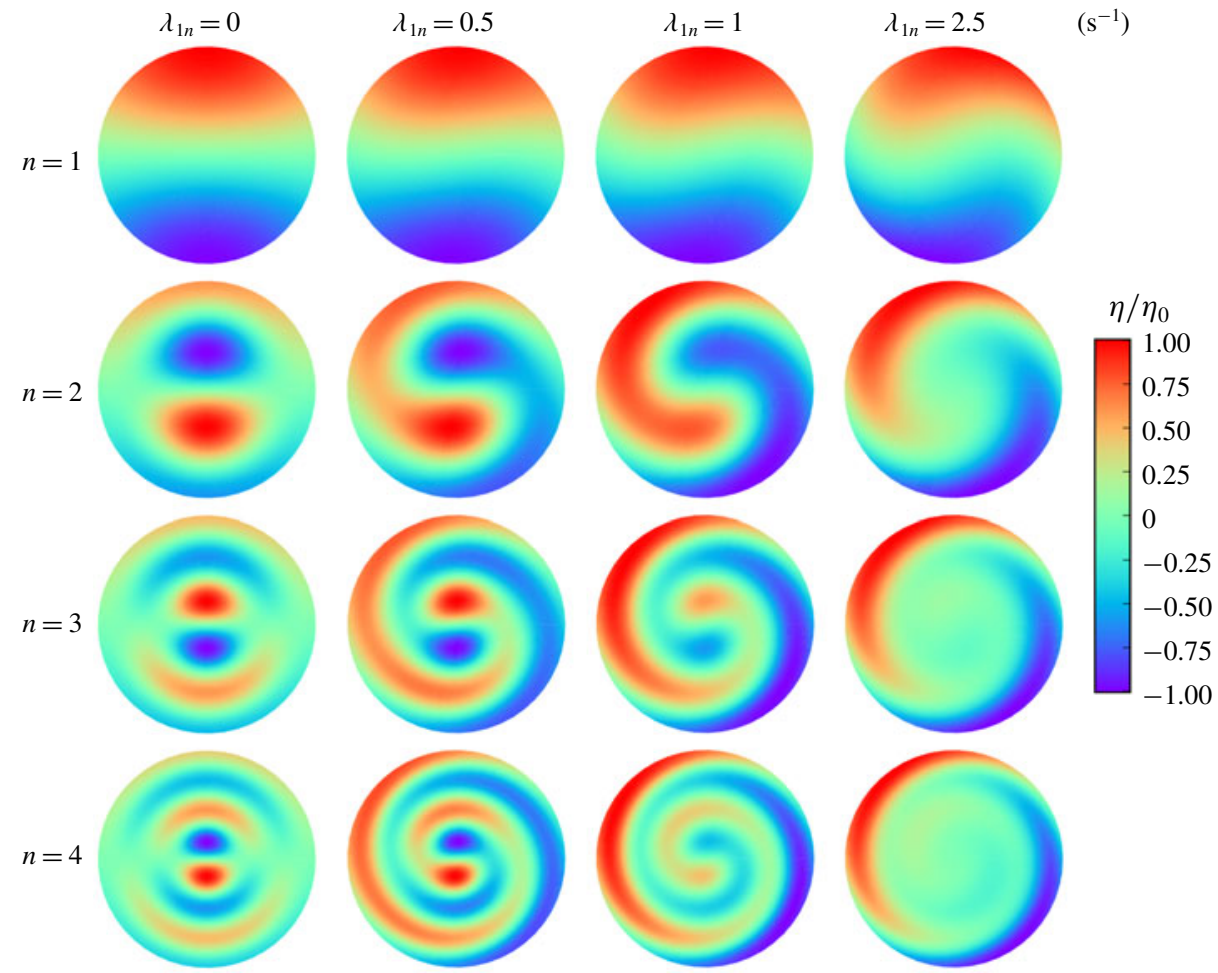

FIGURE 2. Normalised interface elevations $\eta(r, \varphi) / \eta_{0}$ for the first four wave modes $n=$ $(1,2,3,4)$ for four different damping rates $\lambda_{1 n}=(0,0.5,1,2.5) \mathrm{s}^{-1}$. The modes were calculated at corresponding resonance frequencies $\Omega=\omega_{1 n}$ using (2.13).

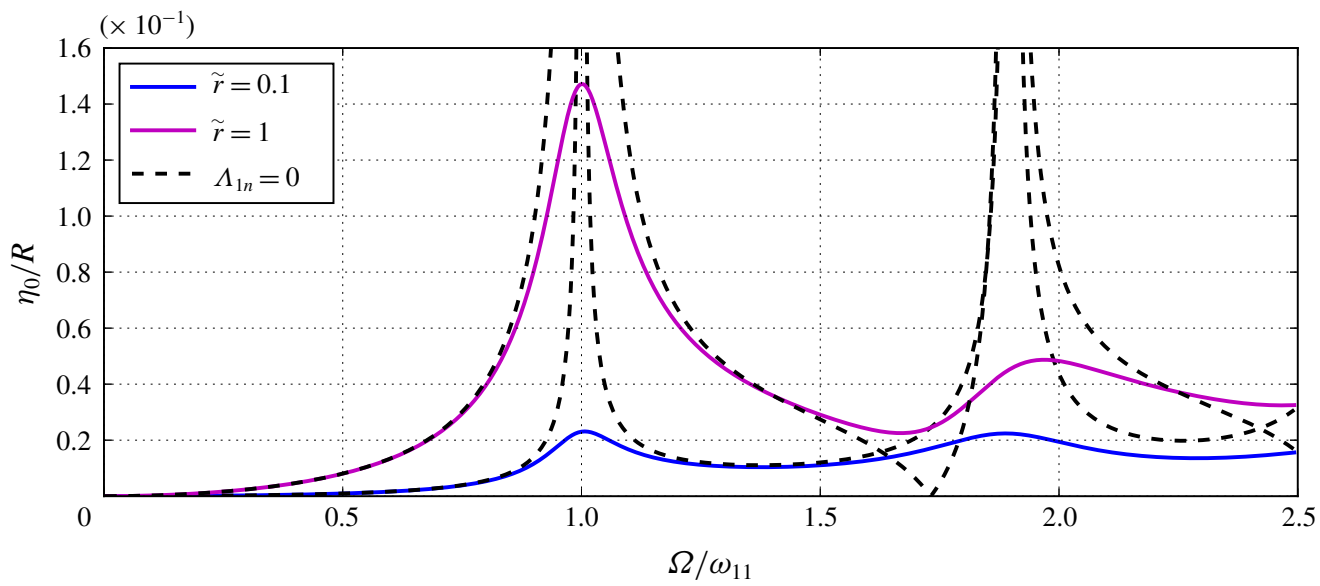

FIGURE 3. Predicted maximum interface elevation $\eta_{0} / R$ for the default parameters given in table 1 as a function of the forcing frequency $\Omega / \omega_{11}$ evaluated near the centre $\tilde{r}=$ 0.1 (blue) and at the sidewall $\tilde{r}=1$ (magenta). The dashed black curves show the corresponding inviscid solutions given by Reclari et al. (2014). 
eigenfrequencies are avoided and the evolution of (linear) resonant wave amplitudes can now be predicted. The resonant amplitudes at the sidewall are considerably decreased for the second mode, while the peak amplitudes at $\tilde{r}=0.1$ remain almost constant. This behaviour underlines the tendency for the highest wave crests to become more and more concentrated near the centre for increasing wave modes, a phenomenon completely missing in the inviscid models. In addition, the inviscid model predicts zero amplitudes for $\tilde{r}=1$ at a singular point between both resonance peaks. This is another non-physical artefact caused by the disregard of damping. The inclusion of damping has markedly improved the predictive power of the model. Both theories coincide for non-resonant frequencies around the first eigenfrequency, while only the damped model can properly describe the linear wave dynamics close to resonance and close to the turnover frequencies between two modes. Both models entirely disagree around the second resonance, because for this example the system is already overdamped such that non-resonant frequencies are also affected.

We now look at the dependence of the resonance dynamics on the different dimensionless numbers (2.1), whereby we focus on the first peak, most relevant in practice. Figure 4 shows the maximum dimensionless wave amplitude $\eta_{0} / R$ at the sidewall $\tilde{r}=1$ versus the Froude number $F r$ for six different parameter variations around the magenta coloured default case (table 1). The lower layer height $h_{2}$ was varied in figure $4(a)$ to modify the aspect ratio $H_{2}$. Two trends can be identified in this plot: the peak amplitudes decrease with decreasing $H_{2}$ and concurrently the eigenfrequencies are reduced. The first phenomenon reflects the strong aspect ratio dependency of the damping rate (A 6). When the interface is located at the centre of the cylinder sufficiently far away from the top and the bottom walls, viscous damping mainly arises at the sidewall. Whereas, when the interface approaches the bottom wall, shearing there causes higher dissipation, which results in lower peak amplitudes. The second effect is easily explained by the dispersion relation (2.9), revealing that gravity waves oscillate slower in shallow layers, a well-known result.

Figure $4(b)$ shows the resonance curves under variation of the viscosity $v_{2}$ to modify the Reynolds number $R e_{2}$. The peak amplitudes are reduced similar to figure $4(a)$ with decreasing $R_{2}$ since the damping (here caused near the sidewalls) grows with $\sim R e_{2}^{-0.5}$. In contrast, the resonance frequencies are almost unaffected. All curves match for the smallest $F r$ and the Reynolds number affects only a window around resonance. This window is slightly expanded on lowering $R e_{2}$ until the overdamped regime is approached for $R e_{2}=10$. Here, damping affects the amplitudes also at non-resonant frequencies. However, for $R e_{2} \lesssim 10$ we start to violate condition (2.40) and the wave elevation at the tank wall is expected to be even more suppressed by ingrowing boundary layers.

The Bond number $B o$ is modified in figure $4(c)$ by varying the interfacial tension $\gamma$. This figure captures the transition from gravity to gravity-capillary waves manifested mainly in increasing resonance frequencies. Moreover, both the height and width of the resonance peak are enlarged due to the coefficient $2 \operatorname{Fr}\left(1+\epsilon_{11}^{2} / B o\right)^{-1}$ from (2.25).

In figure $4(d)$ we have varied the density $\rho_{2}$ to modify the Atwood number $A$ (Bo is weakly affected as well). The Atwood number has a large impact on both the eigenfrequency and the maximum amplitude. It can be understood as a measure of the influence of the upper layer on the wave dynamics. In other words, it describes the transition from one-layer surface waves to two-layer interfacial waves, the latter experiencing a weaker gravity force $\sim\left(\rho_{2}-\rho_{1}\right) g$. The plot underlines again that our model is best suited to interfacial waves (cf. Horstmann et al. 2019). In practice, $A$ is small for most common two-layer stratifications and a small $A$ reduces the 


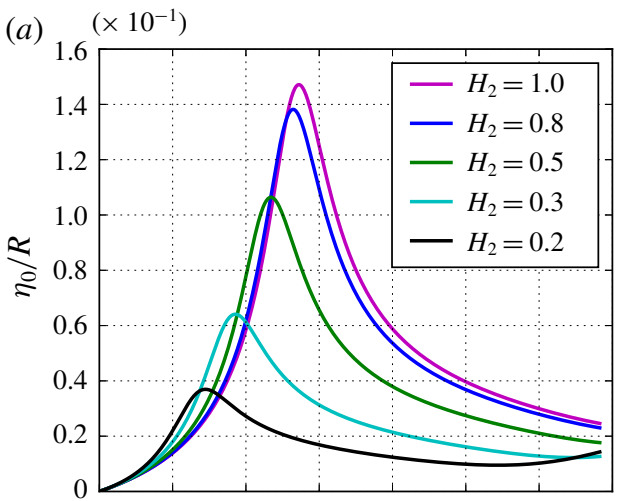

(b)

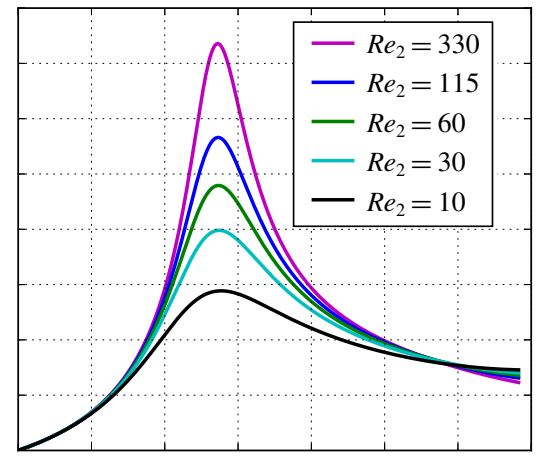

(c) $2.5\left(\times 10^{-1}\right)$

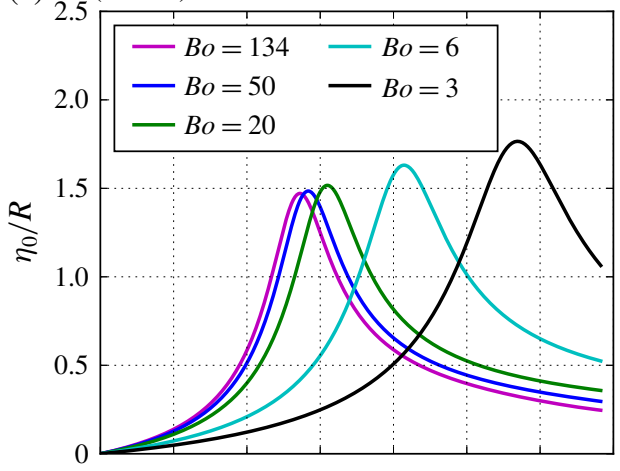

(d)

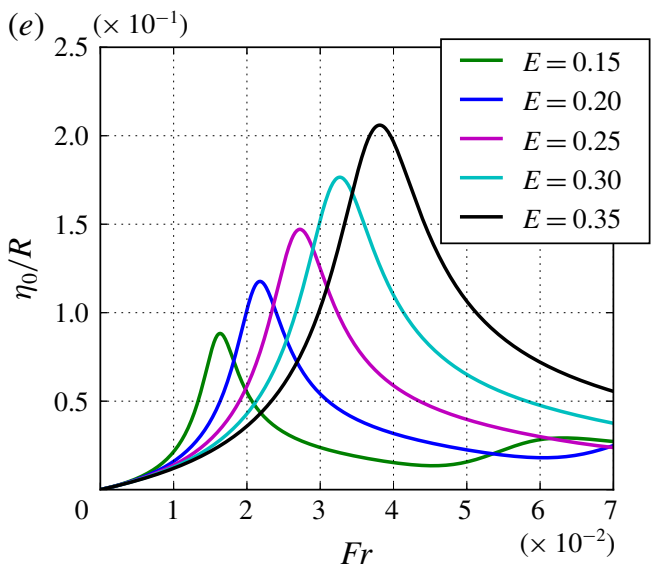

$(f)$
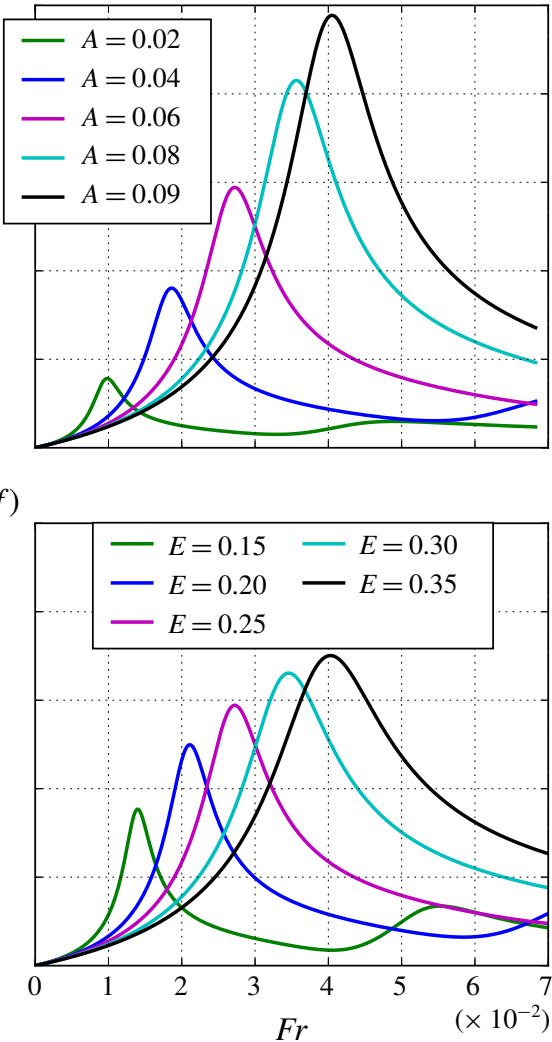

FIGURE 4. Predicted wave amplitude responses around the first resonance frequency at $\tilde{r}=1$ in dependence of the Froude number with different varying parameters around the default case given in table 1 . The parameters $h_{2}(a), v_{2}(b), \gamma(c), \rho_{2}(d), d_{s}(e)$ and $R(f)$ were varied in each plot to modify the dimensionless numbers $H_{2}(a), \operatorname{Re}_{2}(b), B o(c)$, $A(d), E(e)$ and $E(f)$ while keeping constant all other parameters.

wave amplitudes, so that even resonant waves are likely to remain linear for typical shaking conditions. Free-surface waves imply $A=1$, a magnitude that would cause wave breaking in this case long before the eigenfrequency, around which damping has an impact, could be reached. 
The influence of applying different shaking diameters $d_{s}$ is shown in figure 4(e), both the eccentricity $E$ as well as the Froude number $F r$ are affected. Peak amplitudes increase linearly with $E$, as predicted by (2.31). A general linear dependence of $E$ on the wave amplitudes and velocities was already reported by Bouvard et al. (2017). The Fr shifts are linear as well simply due to the scaling $F r \sim d_{s}$ in (2.1).

Finally, the cylinder radius $R$ was varied in figure $4(f)$, influencing multiple numbers $E, R_{1}, R_{2}, H_{1}, H_{2}$ and $B o$. Here, the wave slopes are reduced for increasing $R$ and shifted to smaller $F r$, which might appear somewhat counterintuitive since damping rates are smaller in large containers. However, the eigenfrequencies are also reduced in larger tanks by which the driving force is effectively alleviated at resonance. This is an effect that overcompensates the diminished damping rates. This example shows that our linear approach can also be potentially relevant for large-size applications.

\section{Comparisons with experiments}

We compare our theory in this section with three different and independent experiments: (i) the linear wave amplitudes and phase shifts of the multi-layer interfacial wave experiment by Horstmann et al. (2019); (ii) the weakly nonlinear velocity measurements conducted by Bouvard et al. (2017); and (iii) the scaling laws and the nonlinear phase transition presented by Weheliye et al. (2013), Ducci \& Weheliye (2014) and Weheliye et al. (2018).

\subsection{Two-layer wave elevation comparisons with Horstmann et al. (2019)}

The multi-layer orbital sloshing experiment by Horstmann et al. (2019) was designed with the intention to imitate the wave dynamics excited by the metal pad roll instability in liquid metal batteries (Weber et al. 2017; Horstmann et al. 2018). Controlling the contact line dynamics was the major experimental difficulty. For most liquid combinations Horstmann et al. (2019) observed entirely or partially fixed contact lines, with the latter additionally subject to a contact angle hysteresis. Complex contact line dynamics affects both the wave modes and the damping rates and is not covered in our model, however, recently, Viola \& Gallaire (2018) developed a theoretical framework treating all these effects. However, the particular combination of Paraffinum Perliquidum (PP) $\left(\rho_{1}=846 \mathrm{~g} \mathrm{~cm}^{-3}, v_{1}=36 \mathrm{~mm}^{2} \mathrm{~s}^{-1}\right)$ overlaying Wacker ${ }^{\circledR}$ AK 35 silicone oil $\left(\rho_{2}=955 \mathrm{~g} \mathrm{~cm}^{-3}, v_{2}=35 \mathrm{~mm}^{2} \mathrm{~s}^{-1}\right)$ fulfilled the idealised boundary condition we have used in this study: no meniscus was visible and the contact line slid almost freely along the sidewalls. Hence, these oils are a promising choice for comparisons.

Horstmann et al. (2019) observed that the measured resonance frequencies differed from the theoretical natural eigenfrequencies (2.9) in many stratifications. The inviscid resonance curves appeared shifted relative to the measured ones. The reason for these deviations were, on the one hand, the fixed contact lines in oil|water stratifications that increased the peak frequency with respect to (2.9). On the other hand, a similar shift was also observable in many oil|oil combinations as for PP|AK 35, caused by partial mixing at the interface. Partial mixing led to a temporally increasing reduction of the effective density difference $\rho_{2}-\rho_{1}$, eventually lowering the measured peak frequency with respect to (2.9).

We can nevertheless compare the theory with the experiments when using the measured resonance frequency in (2.25) instead of the theoretical frequency (2.9). In figure 5(a), resonance curves calculated accordingly are compared with the PP $\mid \mathrm{AK} 35$ 

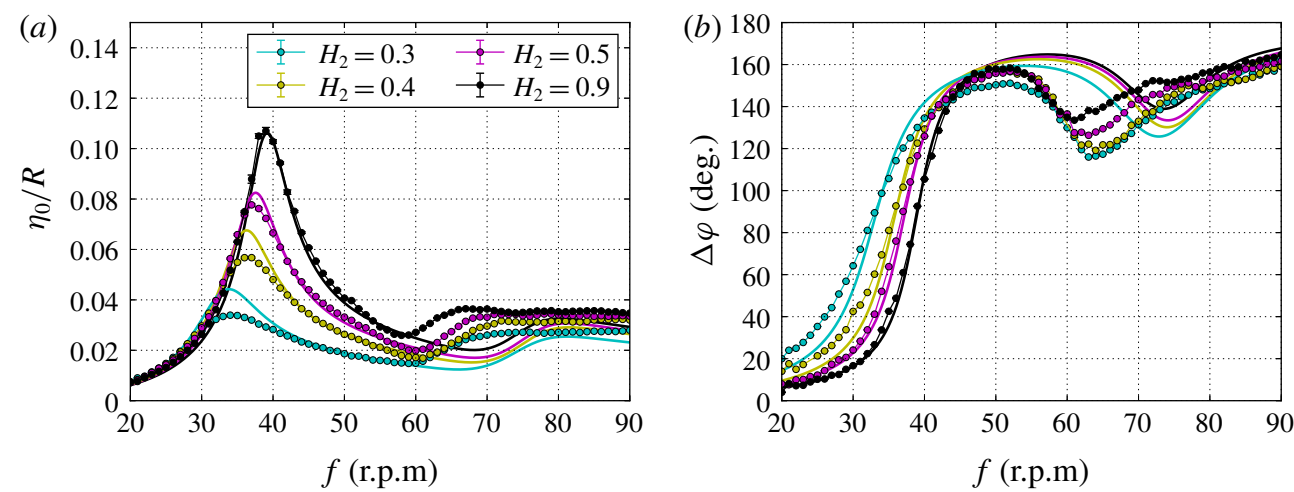

FIGURE 5. (a) Experimental resonance curves (circular markers) of paraffin oil|silicon oil interface elevations at the position $\tilde{r}=0.84$ presented in Horstmann et al. (2019) for four chosen values of $\mathrm{H}_{2}$ in comparison with the theoretical prediction (2.25). Resonance curves are depicted by the dimensionless local wave amplitudes as a function of the shaking frequency $f=\Omega / 2 \pi$. (b) Phase shift $\Delta \varphi$ between wave and shaker for the same measurements. The theoretical phase shifts were calculated using (2.27).

measurements of Horstmann et al. (2019) for four representatively selected values of $\mathrm{H}_{2}$. A small cylinder of radius $R=5 \mathrm{~cm}$ and a shaking diameter of $d_{s}=2.5 \mathrm{~cm}$ were used, and the interface elevation was measured at a fixed position $r=4.2 \mathrm{~cm}$. The theoretical prediction coincides well with the experimental data around the first resonance $f \lesssim 60$ r.p.m.; only the amplitudes close to the peaks are slightly overestimated for the shallow cases. This was expected since additional dissipation mechanisms (e.g. based on interface contamination or the contact line dynamics) are not covered by the theoretical damping rate (2.17). They are, however, unavoidable in the experiments. The direct comparison between measurements and theory in Herreman et al. (2019) demonstrates that the theory slightly underpredicts the measured damping rates. Nevertheless, the first peak is fairly well captured even for the highly damped case $H_{2}=0.3$, where, for $f \gtrsim 38$ r.p.m., the existing inviscid theory largely fails. The second peak is completely damped out in our experiments. This behaviour is reflected in the theory, although there is a disagreement in the range 60 r.p.m. $\lesssim f \lesssim 80$ r.p.m. We observed in the experiment that high excitation frequencies $f \gtrsim 60$ r.p.m. lead to interface ripples, probably causing substantial increase of the system's dissipation and explaining the mismatch here. Qualitatively, the theory can describe how amplitude saturations can result from the superposition of higher overdamped wave modes.

Complementarily, figure $5(b)$ shows the theoretical and experimental phase shifts $\Delta \varphi$ for the same measurements. As for the amplitudes, the phase shifts around the first resonance are well described by our model, while the experimentally observed phase jumps for $H_{2}=0.4$ and $H_{2}=0.3$ are somewhat smoother than predicted due to the slightly underestimated damping discussed before. For higher frequencies, the theoretical phase shifts overshoot the measured curves. However, here, the theory (2.27) qualitatively reveals why a phase shift of $\Delta \varphi=180^{\circ}$ is never reached. It predicts individual phase shifts for every eigenfrequency which are progressively smoothed out by increased damping. If the damping is strong enough, as in the present case, the second mode causes a reversed phase shift before a full out-of-phase flow can be developed. Eventually, the superposition of strongly smoothed phase transitions at higher modes yields the nearly linear growth we observe in $5(b)$ at the 
highest frequencies. Hence, our comparisons show that the model captures all the relevant physics causing this damped resonance dynamics. Because it is challenging to control the experimental conditions, the experimental damping rates are always slightly underestimated by the theory. However, all measured resonance and phase curves shown here can be almost perfectly reproduced by the theory, if fitted values of the damping rates instead of analytical values (2.21) are used. Following this procedure, our model can also be employed to determine linear damping rates by measuring only wave amplitudes or phase shifts. This is particularly useful when damping is high and the exponential decay happens too rapidly for a precise calculation of the decay rates.

\subsection{Velocity comparisons with Bouvard et al. (2017)}

Bouvard et al. (2017) created an orbital sloshing experiment with the aim to investigate the wave-induced mean mass transport in the weakly nonlinear regime. They conducted a series of measurements in a glass cylinder of radius $R=5.12 \mathrm{~cm}$ and height $h_{2}=11.1 \mathrm{~cm}$. Silicon oils with a kinematic viscosity of either $v=50$ or $500 \mathrm{~mm}^{2} \mathrm{~s}^{-1}$ and surface tension of $\gamma=21 \times 10^{-3} \mathrm{~N} \mathrm{~m}^{-1}$ were used as working fluids. Velocity fields were measured by particle imaging velocimetry at different vertical and horizontal light sheet positions. While the study focuses on the mean flow, which is not covered in our approach, other fundamental flow properties such as the wave fields and phase shifts are reported as well. Bouvard et al. (2017) defined the norm of the horizontal velocity at the cell centre $r=0$ and height $z=z_{0}$ as a measure of the wave amplitude

$$
\left|u_{\perp}\right|\left(r=0, z=z_{0}\right)=\sqrt{u_{r}^{2}+u_{\varphi}^{2}} .
$$

Figure 6(a) shows the normalised wave amplitude as a function of the normalised shaking frequency $\Omega / \omega_{11}$ at $z_{0}=-0.23 R$ under excitation with $E=0.057$ for both silicon oils. The theoretical curves were calculated by the potential solution (A $1 b$ ) together with (2.5) in the one-layer limit $\rho_{1}=0$. It becomes clear that our approach does not provide an advantage for $v=50 \mathrm{~mm}^{2} \mathrm{~s}^{-1}$. The damped solution mainly coincides with the inviscid theory and highly overestimates the peak velocities. This is not unexpected since Bouvard et al. (2017) report a hysteresis of the resonant wave dynamics and thus clearly nonlinear behaviour. Here, we exceed the limits of our approach. The nonlinear multimodal theory by Faltinsen, Lukovsky \& Timokha (2016) was recently adapted to orbital sloshing (Raynovskyy \& Timokha 2018b) and allows the first predictions of steady-state sloshing dynamics in this resonant regime. An advantage of our model is visible for $v=500 \mathrm{~mm}^{2} \mathrm{~s}^{-1}$. Here, our theory tends to overestimate the resonant velocities as well. This might be due to the influence of ingrowing boundary layers or additional experimental damping mechanisms not captured by our theory (surface contamination, slight contact line hysteresis). However, for frequencies higher than the resonance frequency, the measured wave velocities agree better with our theory than with the inviscid model.

Generally, it is known that potential models can more accurately predict interface elevations (especially for higher amplitudes) than velocity fields. The latter are influenced to a larger degree by boundary layers and secondary flow structures not captured in potential approaches (Ibrahim 2005). This observation is supported by figure $6(b)$, showing the corresponding phase shifts for both oils. Our model (2.28) can predict the frequency-dependent shift well even for $v=50 \mathrm{~mm}^{2} \mathrm{~s}^{-1}$, although 

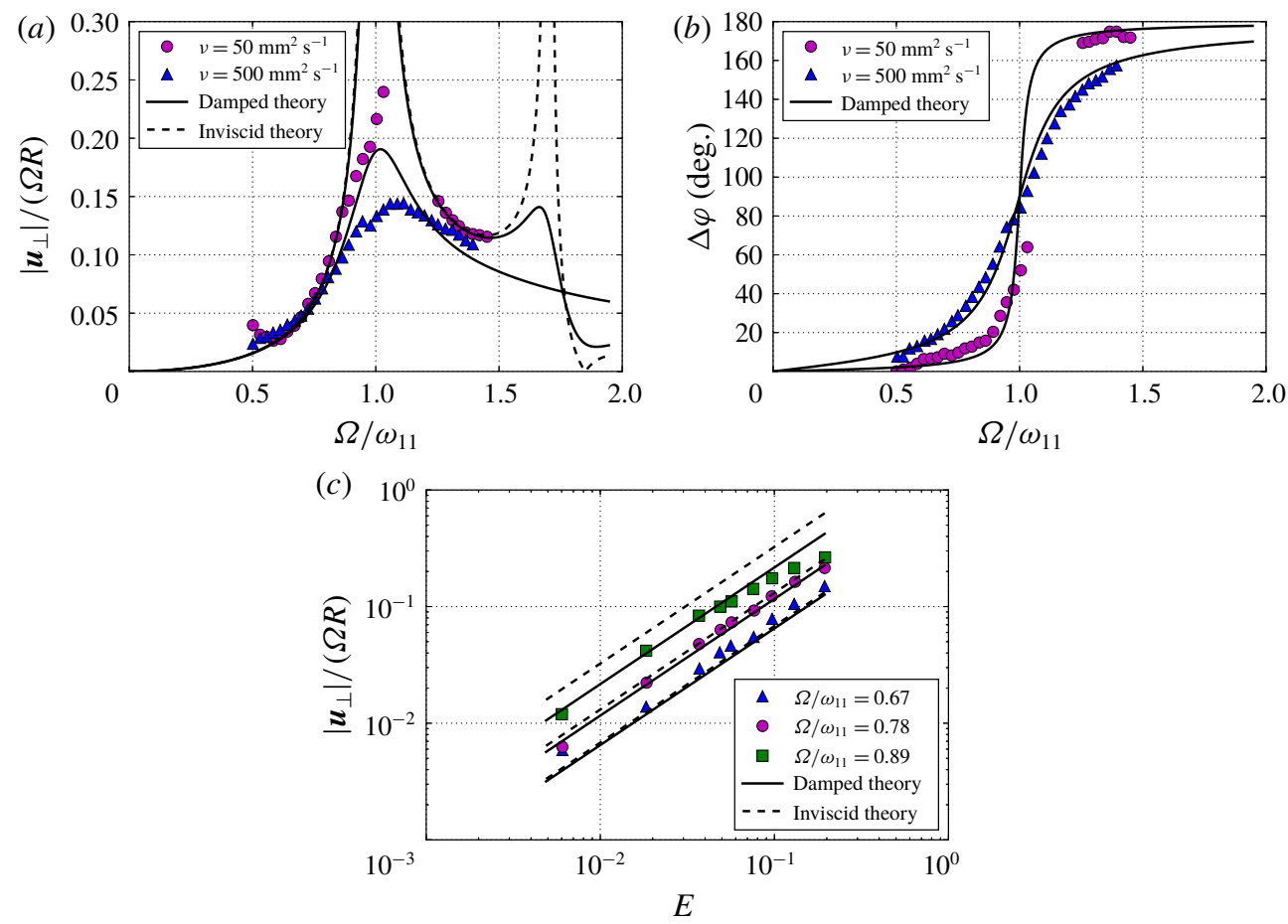

FIGURE 6. (a) Normalised velocity amplitude measured at $r=0, z_{0}=-0.23 R$ as a function of the dimensionless shaking frequency $\Omega / \omega_{11}$ for both silicon oils and $E=0.057$. The markers show the measurements conducted by Bouvard et al. (2017), the black line our theoretical prediction due to the potential solution $(2.12 b)$ and the dashed line the inviscid prediction by Reclari et al. (2014). (b) Phase shifts as a function of $\Omega / \omega_{11}$ for the same measurements. (c) Normalised velocity amplitude as a function of $E$ of the viscous silicon oil $\left(v_{2}=500 \mathrm{~mm}^{2} \mathrm{~s}^{-1}\right)$ for three different shaking frequencies.

it failed to predict the resonant velocities. The phase shift of the centre velocity coincides with the phase shift of the surface elevation, which seems to be much more robust towards effects of (weakly) nonlinear waves and secondary rotational flows.

Bouvard et al.'s (2017) measurements of the horizontal centre velocity obtained with the viscous silicon oil $v=50 \mathrm{~mm}^{2} \mathrm{~s}^{-1}$ for varying shaking diameters $E$ at three different shaking frequencies are shown in figure $6(c)$. A linear scaling between the velocity and $E$ becomes apparent. For the two measurement series $\Omega / \omega_{11}=0.67$ and 0.78 conducted at frequencies sufficiently below the resonance, Bouvard et al. (2017) found a good agreement with the inviscid theory. The damped predictions scarcely deviate from the inviscid ones and the effect of damping is weak as discussed before. But for the measurement $\Omega / \omega_{11}=0.89$, which is inside the resonant regime, our damped model coincides considerably better. The curve fits the first three or four measurements, until higher forcing parameters $E$ excite a nonlinear dynamics, manifested in an incipient saturation.

\subsection{Scaling law and nonlinear out-of-phase flow prediction in comparison with Weheliye et al. (2013)}

Recently, Weheliye et al. (2013), Ducci \& Weheliye (2014) and Weheliye et al. (2018) have made significant progress in identifying and understanding the fluid mechanics 
of OSBs with a focus on the mixing dynamics. Most OSBs operate in strongly nonlinear regimes, which precludes comparison of our theoretical findings. However, these works reported two different physical effects of high practical relevance, which can be described by our theory under certain assumptions discussed and verified below.

A main result of Weheliye et al. (2013) is the existence of a linear scaling law

$$
\frac{\eta_{0}}{R}=\alpha_{0} F r
$$

very useful for estimating surface elevations in OSBs. The constant $\alpha_{0}$ is reported to depend on the working fluid. Its value is $\alpha_{0}=1.4$ for water, while lower values were measured for silicon oils of higher viscosity (Ducci \& Weheliye 2014). The scaling law was experimentally well verified for small and moderate Froude numbers $\mathrm{Fr} \lesssim 0.3$ and frequencies much smaller than the resonance frequency, a regime that our theory should be able to describe. However, our solution (2.25) does not contain any linear scaling, except for the asymptotic limit of extremely small shaking frequencies $F r \ll E$. In this limit, the surface elevation is determined solely by the balance between the centrifugal acceleration and the gravitational force. Figure 7(a) shows solution (2.25) (lines) together with the $\eta_{0} / R$ values (triangles) measured by Weheliye et al. (2013) versus $\mathrm{Fr}$ for different $E$ and $H$. Weheliye et al. (2013) used two glass cylinders of radii $R=5 \mathrm{~cm}$ and $6.5 \mathrm{~cm}$ partially filled with water of different depths. Weheliye et al.'s (2013) scaling law with $\alpha_{0}=1.4$ is drawn in figure 7(a) as the dashed black line. Since all $E$ cases can be described by the potential model (except for the highest $\mathrm{Fr}$, where the wave is becoming nonlinear), there is no linear dependency. Weheliye et al. (2013) have measured a series of resonance curves (compare with figure 4e) which appear to be linear in superposition. There is in fact only a linear dependency for the smallest $\mathrm{Fr}<0.05$, which can be described by the flat disc solution $\eta_{0} / R=F r$ (dash-dotted black line) contained in (2.32). For the sake of clarity, we have omitted the $H$ cases in figure $7(a)$; however, they fit to the potential model as well.

For Weheliye et al.'s (2013) set-up, solution (2.25) is not affected by damping rates of liquids with $v \lesssim 1000 \mathrm{~mm}^{2} \mathrm{~s}^{-1}$ because all measurements were conducted in the non-resonant regime long before the first resonance is reached. As a result, equation (2.25) predicts the same surface elevations as the inviscid model of Reclari et al. (2014). This contradicts Ducci \& Weheliye's (2014) assumption that the proportionality constant $\alpha_{0}$ depends on the fluid viscosity. Based on $\eta_{0} / R-F r$ measurements with oils of different viscosity, Ducci \& Weheliye (2014) found the following scaling law for $\alpha_{0}$

$$
\alpha_{0}(v)=\alpha_{0 w}\left(\frac{v}{v_{w}}\right)^{-0.0256},
$$

where $\alpha_{0 w}$ and $v_{w}$ denote the proportionality constant and the viscosity of water. The corresponding fitting curve is plotted in figure $1(b)$ of Ducci \& Weheliye (2014). In our opinion, this law does not properly describe the underlying physics for two reasons: first, it predicts a strong dependency on the viscosity for low-viscosity fluids close to water, while the dependency is very weak for high-viscosity liquids $v \gtrsim 100 \mathrm{~mm}^{2} \mathrm{~s}^{-1}$. This is the exact opposite of what is predicted by our wave theory. Second, the decay of $\alpha_{0}$ is extremely weak in the limit of high $v$ so that one obtains, e.g. for bitumen $\left(v \sim 1 \times 10^{11} \mathrm{~mm}^{2} \mathrm{~s}^{-1}\right)$ a constant of $\alpha_{0} \approx 0.7$. Consequently, for 

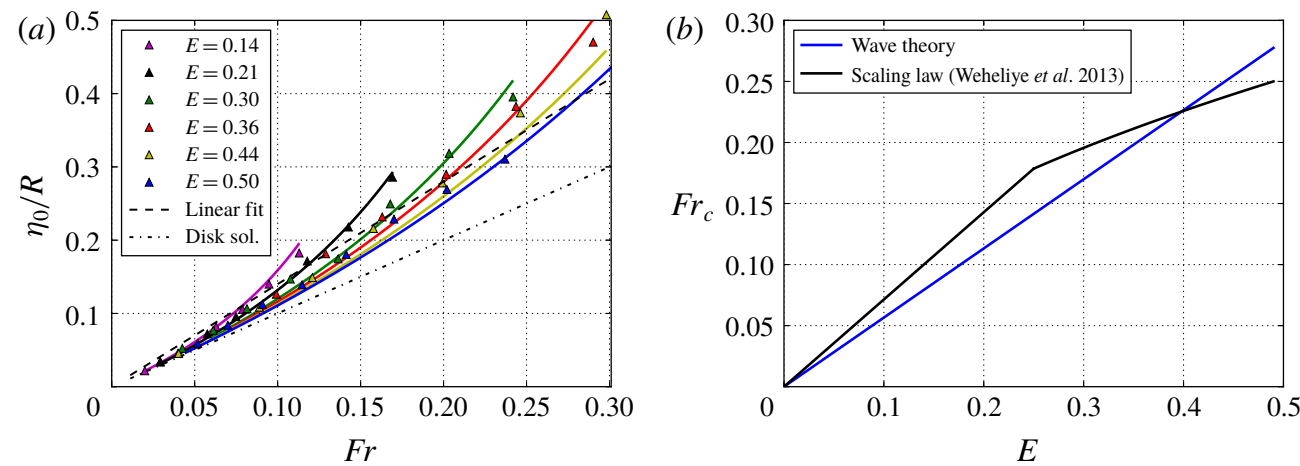

FIgURE 7. (a) Replot of the $E$ cases shown in (a) of Weheliye et al. (2013). Normalised surface elevations are plotted as a function of $F r$. The markers show the measurements and the coloured lines represent the corresponding analytical predictions (2.25). In addition, the dashed black line marks the linear fit (4.2) with $\alpha_{0}=1.4$ and the dash-dotted black line shows the disc solution $\eta_{0} / R=F r$. (b) Predictions of critical $F r$ numbers initiating the nonlinear out-of-phase flow transition as a function of $E$ for $H=1$ due to our wave theory-based prediction (4.6) (blue line) as well as the empirical law (4.5) presented in Weheliye et al. (2013) (black line).

bitumen, the law predicts sloshing with half the amplitude of water, an unlikely result.

In our opinion, the concrete value of $\alpha_{0}$ is mainly determined by the cutoff points chosen for the single measured $E$ and $H$ cases. If the highest $F r$ measurement is removed for every $E$ case in figure 7(a), one would obtain a considerably lower fitting constant $\alpha_{0}$. This can be seen from Ducci \& Weheliye's (2014) figure 1(a) as well, where only small-Fr cases are included, resulting in a reduced constant of $\alpha_{0}=1.23$. Ducci \& Weheliye (2014) attribute this only to the higher viscosity of the employed liquid. However, except for the amplitude-viscosity dependency that we deem to be unphysical, equation (4.3) is a valuable estimate for the design of OSBs, but one has to know its limits.

Weheliye et al. (2013) explain the deviation of the highest $F r$ measurements from the fit with differences in surface waviness. The surface is flat for low Fr, but gets wavier with increasing $\mathrm{Fr}$. This necessitates the use of an average of the local wave slopes, thereby introducing errors. Our interpretation of this observation is different. That the data points start to deviate from the fit as soon as the surface becomes wavy is in line with our theory of the disc-wave transition. In $\S 2.4$ we have derived an expression for the critical $\mathrm{Fr}$ number necessary to induce a certain waviness $\Upsilon$

$$
F r_{\text {wave }}(\Upsilon)=\frac{E \epsilon_{11} \tanh \left(\epsilon_{11} H\right)}{2\left(\epsilon_{11}^{2}-1\right)^{-1} \Upsilon^{-1}+1}
$$

We found that all presented $E$ cases escape as soon as a waviness of $\Upsilon=40 \%$ is reached. All $E$ cases were conducted with $H=1$. Equation (4.4) predicts the critical $F r_{\text {wave }}(40 \%)=\{0.08,0.12,0.17,0.2,0.25,0.28\}$ for the plotted cases $E=\{0.14,0.21,0.3,0.36,0.44,0.5\}$. Figure $7(a)$ reveals that these $F r_{\text {wave }}$ values coincide with the intersection points of the linear fit with the resonance curves. The evolution of a wavy-shaped surface is indeed a good indicator for a slowly emerging resonance here causing the nonlinear increases of the wave amplitudes. 
Weheliye et al. (2013) concluded correctly that $\alpha_{0}$ is always greater than unity because it takes into account an extra inertial force, besides the centrifugal acceleration introduced by the shaker. This extra force is caused by the movement of the liquid itself; however, it is not constant and depends on the waviness and therefore also on $F r$. Exactly this point was overlooked in the studies before.

To conclude, equation (4.4) can be used to determine the limits of the scaling law (4.2). Equation (4.2) provides a good estimate for surface elevations as long as $F r \lesssim$ $F r_{\text {wave }}(\Upsilon)$. An empirical parameter in form of the waviness $\Upsilon$ remains that depends on the fit. The smaller we choose the value of $\Upsilon$ the more accurate (4.2) becomes. But physically there is no linear dependency in the $\mathrm{Fr}$ regime considered and we recommend using (2.25) directly for more precise calculations.

Based on the preceding paragraphs, we can draw some conclusions regarding the out-of-phase flow frequently observed in OSBs. Such a flow is different from the linear phase shifts considered, e.g. by Bouvard et al. (2017) and Alpresa et al. $(2018 a)$. The linear phase transition always arises at resonance and is smoothed out by internal damping forces. The phase transition observed in many OSB studies (Büchs et al. 2000; Weheliye et al. 2013; Ducci \& Weheliye 2014; Klöckner et al. 2014; Thomas et al. 2017; Rodriguez et al. 2018; Weheliye et al. 2018) is physically different and caused by nonlinear secondary flows. Weheliye et al. (2013) demonstrated the existence of two counter-rotating toroidal vortices close to the free surface, which are not predicted by our linear theory. With increasing $F r$, these vortices move towards the walls and shrink until they disappear. This initiates the flow transition to the out-of-phase regime. Weheliye et al. (2013) observed that, simultaneously, the initially flat free surface becomes wavy, exactly the transformation which we have described using (4.4). Under the proposition that the out-of-phase transition always arises as soon as the flat disc is destabilised, we can also predict this nonlinear flow transition since the flat disc is always a linear solution captured by our theory. Weheliye et al. (2013) found the following empirical law for the critical $\mathrm{Fr}$ number, above which the out-of-phase flow is expected to arise:

$$
F r_{c}= \begin{cases}\frac{1}{2 \alpha_{0}} H \sqrt{E}, & \text { if } H<2 \sqrt{E}, \\ \frac{1}{\alpha_{0}} E, & \text { if } H>2 \sqrt{E} .\end{cases}
$$

On the basis of (4.4), we propose

$$
F r_{c}=\frac{E \epsilon_{11} \tanh \left(\epsilon_{11} H\right)}{2\left(\epsilon_{11}^{2}-1\right)^{-1} \Upsilon^{-1}+1}
$$

as an alternative prediction. In direct comparison, it becomes apparent that (4.6) contains the predicted $H$ scaling of both cases in (4.5) as asymptotic limits: a linear scaling $F r_{c} \sim H$ for $H \ll 1$ and a saturation $F r_{c}=$ const. for $H \gg 1$. Hence, the $H$ dependence observed by Weheliye et al. (2013) can be, at least partially, explained by the transition from the shallow water to the deep water wave regime. Both laws are graphically compared in figure $7(b)$ as a function of $E$ with a constant aspect ratio of $H=1$. Our criterion (4.6) also involves the empirical waviness $\Upsilon$ (here set to $\Upsilon=40 \%$, comparable with $\alpha_{0}=1.4$ ). It can be seen that (4.6) is very close to the established empirical law (4.5). Since the out-of-phase transition is not sharp and proceeds gradually, it can be well explained by the disc-to-wave transition alone. 
However, the $\sim \sqrt{E}$ scaling for small $H<2 \sqrt{E}$ in (4.5) is not captured by (4.6). For larger $E$, equation (4.6) starts to overestimate critical $\mathrm{Fr}$ numbers in comparison with (4.5). In this case, the flow transition is not caused by the motion of the free surface alone, which drives the toroidal vortices. Weheliye et al. (2013) found that for $2 \sqrt{E}>H$ the vortices are steadily pushed towards the bottom wall. This initiates the out-of-phase transition in a way similar to the interaction with the sidewalls for $2 \sqrt{E}<H$. In this case, waviness alone is not a sufficient criterion anymore and we cannot draw any further conclusions from our model. However, we can physically explain and support all other empirical scalings in (4.5) by relating the linear fitting constant $\alpha_{0}$ to the specific waviness of the free surface.

\section{Concluding remarks}

By complementing linear sloshing theory with viscous damping rates recently calculated by Herreman et al. (2019), we have derived analytical formulas for wave amplitudes, phase shifts and fluid velocities of two- and one-layer gravity-capillary waves in orbitally shaken cylinders. In contrast to the previous inviscid theory of Reclari et al. (2014), our model avoids singularities and can predict the evolution of interface elevations over the different eigenfrequencies, as long as the wave remains in the linear regime. Resonance dynamics was discussed utilising eight dimensionless numbers, which revealed multiple different mechanisms that affect the wave amplitudes. Weak viscous damping reduces only the highest amplitudes around resonance, while stronger damping affects the amplitudes of all shaking frequencies, when the system becomes overdamped. The strong amplitude reduction observed by Horstmann et al. (2019) for interfaces approaching the top or bottom wall could be adequately quantified. In addition, the theory comprises the transition from gravity to capillary waves characterised by the Bond number as well as the transition from one-layer to two-layer systems determined by the Atwood number. As an intriguing result, our model predicts novel spiral wave patterns in the presence of damping. They are caused by a relative twisting of the nodal cycles that breaks the symmetry. Linear spiral wave solutions are rare in physical systems and offer a promising basis for further research.

For validation, we have compared our theory with three independent experiments by Horstmann et al. (2019), Bouvard et al. (2017) and Weheliye et al. (2013). Within the limits of the theory, predictions are in very good agreement with all experiments, provided that the predicted resonance frequencies are slightly adjusted to the measured values. The saturation of the amplitude and the phase of the first eigenfrequency discovered by Horstmann et al. (2019) can be explained by the superposition of overdamped higher wave modes. The phase shifts measured by Bouvard et al. (2017) fit to the predictions, despite the fact that some measurements were conducted in the weakly nonlinear regime. It was further demonstrated that the linear scaling law $\eta_{0} / R=\alpha_{0} F r$ of Weheliye et al. (2013) results from a combination of disjoint resonance curves. Consequently, the postulated linear dependency is just an approximation for sufficiently small Froude numbers where free surfaces behave more like tilted planes than as curved waves. Based on this finding, we derived an expression for the nonlinear out-of-phase flow transition that is important for the mixing efficiency in orbitally shaken bioreactors. A comparison with the empirical law of Weheliye et al. (2013) revealed that an incipient phase shift is triggered solely by the transformation of the free surface from a plane disc to a curved wave, as long as the surface is sufficiently far away from the ground wall. 
These findings open different perspectives for further research. Our linear approach quantifies the resonance dynamics in two-layer interfacial wave systems with good accuracy. However, one-layer systems, such as shaken bioreactors, typically induce nonlinear wave dynamics near the resonance frequencies. For those systems, nonlinear models like the one by Raynovskyy \& Timokha (2018b) are needed. A classification of different phase shift regimes is yet to be done. While the mechanisms of linear and nonlinear phase transitions are essentially understood, in which parameter ranges different kinds of phase shifts can be triggered remains to be settled. An experimental verification and an in-depth study of the spiral waves predicted for highly damped systems would support the theory and seems worthwhile from the perspective of basic research.

\section{Acknowledgements}

We acknowledge C. Nore, N. Weber and F. Stefani for fruitful discussions on several topics related to this work. We are particularly thankful to J. Tithof for his careful reading of the manuscript.

\section{Declaration of interests}

The authors report no conflict of interest.

\section{Appendix A. Dimensionless wave solutions and damping rates}

Flow potentials:

$$
\begin{aligned}
& \frac{\phi_{1}(\tilde{r}, \varphi, \tilde{z}, \tilde{t})}{\Omega R^{2}}=\sum_{n=1}^{\infty} \frac{2 F r}{\left(1+\frac{\epsilon_{1 n}^{2}}{B o}\right)} \frac{\cosh \left(\epsilon_{1 n}\left(\tilde{z}-H_{1}\right)\right)}{\sinh \left(\epsilon_{1 n} H_{1}\right)} \frac{J_{1}\left(\epsilon_{1 n} \tilde{r}\right)}{\epsilon_{1 n}\left(\epsilon_{1 n}^{2}-1\right) J_{1}\left(\epsilon_{1 n}\right)} \\
& \quad \times\left[\frac{\Gamma_{\omega_{1 n}}^{2}}{\left.\left(\Gamma_{\omega_{1 n}}^{2}-1\right)+\frac{\Lambda_{1 n}^{2}}{\left(\Gamma_{\omega_{1 n}}^{2}-1\right)} \sin (\tilde{t}-\varphi)-\frac{\Gamma_{\omega_{1 n}}^{2}}{\frac{\left(\Gamma_{\omega_{1 n}}^{2}-1\right)^{2}}{\Lambda_{1 n}}+\Lambda_{1 n}} \cos (\tilde{t}-\varphi)\right],(\mathrm{A} 1 a)}\right. \\
& \frac{\phi_{2}(\tilde{r}, \varphi, \tilde{z}, \tilde{t})}{\Omega R^{2}}=\sum_{n=1}^{\infty} \frac{-2 F r}{\left(1+\frac{\epsilon_{1 n}^{2}}{B o}\right)} \frac{\cosh \left(\epsilon_{1 n}\left(\tilde{z}+H_{2}\right)\right)}{\sinh \left(\epsilon_{1 n} H_{2}\right)} \frac{J_{1}\left(\epsilon_{1 n} \tilde{r}\right)}{\epsilon_{1 n}\left(\epsilon_{1 n}^{2}-1\right) J_{1}\left(\epsilon_{1 n}\right)} \\
& \quad \times\left[\frac{\Gamma_{\omega_{1 n}}^{2}}{\left(\Gamma_{\omega_{1 n}}^{2}-1\right)+\frac{\Lambda_{1 n}^{2}}{\left(\Gamma_{\omega_{1 n}}^{2}-1\right)} \sin (\tilde{t}-\varphi)-\frac{\Gamma_{\omega_{1 n}}^{2}}{\left(\Gamma_{\omega_{1 n}}^{2}-1\right)^{2}}} \cos (\tilde{t}-\varphi)\right] .
\end{aligned}
$$

Velocity fields:

$$
\frac{u_{r, 1}(\tilde{r}, \varphi, \tilde{z}, \tilde{t})}{\Omega R}=\sum_{n=1}^{\infty} \frac{2 \epsilon_{1 n} F r}{\left(1+\frac{\epsilon_{1 n}^{2}}{B o}\right)} \frac{\cosh \left(\epsilon_{1 n}\left(\tilde{z}-H_{1}\right)\right)}{\sinh \left(\epsilon_{1 n} H_{1}\right)} \frac{J_{1}^{\prime}\left(\epsilon_{1 n} \tilde{r}\right)}{\epsilon_{1 n}\left(\epsilon_{1 n}^{2}-1\right) J_{1}\left(\epsilon_{1 n}\right)}
$$




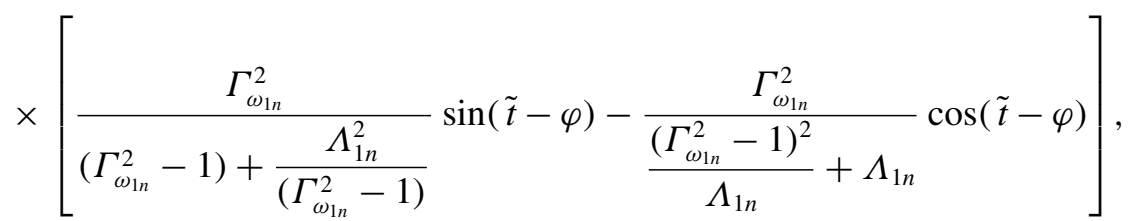

$$
\begin{aligned}
& \frac{u_{\varphi, 1}(\tilde{r}, \varphi, \tilde{z}, \tilde{t})}{\Omega R}=\sum_{n=1}^{\infty} \frac{2 F r}{\left(1+\frac{\epsilon_{1 n}^{2}}{B o}\right)} \frac{\cosh \left(\epsilon_{1 n}\left(\tilde{z}-H_{1}\right)\right)}{\sinh \left(\epsilon_{1 n} H_{1}\right)} \frac{J_{1}\left(\epsilon_{1 n} \tilde{r}\right)}{\epsilon_{1 n} \tilde{r}\left(\epsilon_{1 n}^{2}-1\right) J_{1}\left(\epsilon_{1 n}\right)} \\
& \times\left[-\frac{\Gamma_{\omega_{1 n}}^{2}}{\left(\Gamma_{\omega_{1 n}}^{2}-1\right)+\frac{\Lambda_{1 n}^{2}}{\left(\Gamma_{\omega_{1 n}}^{2}-1\right)}} \cos (\tilde{t}-\varphi)-\frac{\Gamma_{\omega_{1 n}}^{2}}{\frac{\left(\Gamma_{\omega_{1 n}}^{2}-1\right)^{2}}{\Lambda_{1 n}}+\Lambda_{1 n}} \sin (\tilde{t}-\varphi)\right],(\mathrm{A} 2 b) \\
& \frac{u_{z, 1}(\tilde{r}, \varphi, \tilde{z}, \tilde{t})}{\Omega R}=\sum_{n=1}^{\infty} \frac{2 \epsilon_{1 n} F r}{\left(1+\frac{\epsilon_{1 n}^{2}}{B o}\right)} \frac{\sinh \left(\epsilon_{1 n}\left(\tilde{z}-H_{1}\right)\right)}{\sinh \left(\epsilon_{1 n} H_{1}\right)} \frac{J_{1}\left(\epsilon_{1 n} \tilde{r}\right)}{\epsilon_{1 n}\left(\epsilon_{1 n}^{2}-1\right) J_{1}\left(\epsilon_{1 n}\right)} \\
& \times\left[\frac{\Gamma_{\omega_{1 n}}^{2}}{\left(\Gamma_{\omega_{1 n}}^{2}-1\right)+\frac{\Lambda_{1 n}^{2}}{\left(\Gamma_{\omega_{1 n}}^{2}-1\right)}} \sin (\tilde{t}-\varphi)-\frac{\Gamma_{\omega_{1 n}}^{2}}{\frac{\left(\Gamma_{\omega_{1 n}}^{2}-1\right)^{2}}{\Lambda_{1 n}}+\Lambda_{1 n}} \cos (\tilde{t}-\varphi)\right], \\
& \frac{u_{r, 2}(\tilde{r}, \varphi, \tilde{z}, \tilde{t})}{\Omega R}=\sum_{n=1}^{\infty} \frac{-2 \epsilon_{1 n} F r}{\left(1+\frac{\epsilon_{1 n}^{2}}{B o}\right)} \frac{\cosh \left(\epsilon_{1 n}\left(\tilde{z}+H_{2}\right)\right)}{\sinh \left(\epsilon_{1 n} H_{2}\right)} \frac{J_{1}^{\prime}\left(\epsilon_{1 n} \tilde{r}\right)}{\epsilon_{1 n}\left(\epsilon_{1 n}^{2}-1\right) J_{1}\left(\epsilon_{1 n}\right)}
\end{aligned}
$$

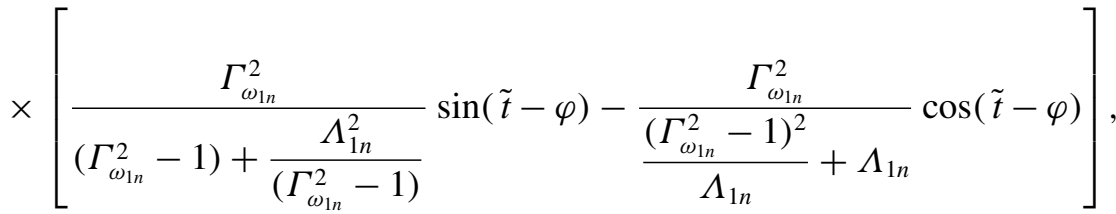

$$
\begin{aligned}
& \frac{u_{\varphi, 2}(\tilde{r}, \varphi, \tilde{z}, \tilde{t})}{\Omega R}=\sum_{n=1}^{\infty} \frac{2 F r}{\left(1+\frac{\epsilon_{1 n}^{2}}{B o}\right)} \frac{\cosh \left(\epsilon_{1 n}\left(\tilde{z}+H_{2}\right)\right)}{\sinh \left(\epsilon_{1 n} H_{2}\right)} \frac{J_{1}\left(\epsilon_{1 n} \tilde{r}\right)}{\epsilon_{1 n} \tilde{r}\left(\epsilon_{1 n}^{2}-1\right) J_{1}\left(\epsilon_{1 n}\right)} \\
& \times\left[\frac{\Gamma_{\omega_{1 n}}^{2}}{\left(\Gamma_{\omega_{1 n}}^{2}-1\right)+\frac{\Lambda_{1 n}^{2}}{\left(\Gamma_{\omega_{1 n}}^{2}-1\right)}} \cos (\tilde{t}-\varphi)+\frac{\Gamma_{\omega_{1 n}}^{2}}{\frac{\left(\Gamma_{\omega_{1 n}}^{2}-1\right)^{2}}{\Lambda_{1 n}}+\Lambda_{1 n}} \sin (\tilde{t}-\varphi)\right], \\
& \frac{u_{z, 2}(\tilde{r}, \varphi, \tilde{z}, \tilde{t})}{\Omega R}=\sum_{n=1}^{\infty} \frac{-2 \epsilon_{1 n} F r}{\left(1+\frac{\epsilon_{1 n}^{2}}{B o}\right)} \frac{\sinh \left(\epsilon_{1 n}\left(\tilde{z}+H_{2}\right)\right)}{\sinh \left(\epsilon_{1 n} H_{2}\right)} \frac{J_{1}\left(\epsilon_{1 n} \tilde{r}\right)}{\epsilon_{1 n}\left(\epsilon_{1 n}^{2}-1\right) J_{1}\left(\epsilon_{1 n}\right)} \\
& \times\left[\frac{\Gamma_{\omega_{1 n}}^{2}}{\left(\Gamma_{\omega_{1 n}}^{2}-1\right)+\frac{\Lambda_{1 n}^{2}}{\left(\Gamma_{\omega_{1 n}}^{2}-1\right)}} \sin (\tilde{t}-\varphi)-\frac{\Gamma_{\omega_{1 n}}^{2}}{\frac{\left(\Gamma_{\omega_{1 n}}^{2}-1\right)^{2}}{\Lambda_{1 n}}+\Lambda_{1 n}} \cos (\tilde{t}-\varphi)\right] .
\end{aligned}
$$


Frequency:

$$
\Gamma_{\omega_{1 n}}=\frac{\omega_{1 n}}{\Omega}=\sqrt{\frac{E}{F r} \frac{2 A \epsilon_{1 n}\left(1+\frac{\epsilon_{1 n}^{2}}{B o}\right)}{\left[(1-A) \operatorname{coth}\left(\epsilon_{1 n} H_{1}\right)+(1+A) \operatorname{coth}\left(\epsilon_{1 n} H_{2}\right)\right]}} .
$$

One-layer damping rate:

$$
\begin{gathered}
\Lambda_{1 n}^{1 L}=\frac{2 \lambda_{1 n}^{1 L}}{\Omega}=\sqrt{\frac{1}{2 R e_{2}}}\left[\frac{\epsilon_{1 n}^{2}+1}{\epsilon_{1 n}^{2}-1}+\frac{2 \epsilon_{1 n}\left(1-H_{2}\right)}{\sinh \left(2 \epsilon_{1 n} H_{2}\right)}\right] \\
+\frac{2}{R e_{2}}\left[2 \epsilon_{1 n}^{2}-\frac{1}{\epsilon_{1 n}^{2}-1}\left(1+\frac{2 \epsilon_{1 n} H_{2}}{\sinh \left(2 \epsilon_{1 n} H_{2}\right)}\right)\right] .
\end{gathered}
$$

Two-layer damping rate:

$$
\begin{aligned}
& \Lambda_{1 n}^{2 L}=\frac{2 \lambda_{1 n}^{2 L}}{\Omega}=\sum_{i=1,2} \sqrt{\frac{1}{2 \operatorname{Re}_{i}}}\left[\frac{(i-1)(1+A)+(2-i)(1-A)}{\left[(1-A) \operatorname{coth}\left(\epsilon_{1 n} H_{1}\right)+(1+A) \operatorname{coth}\left(\epsilon_{1 n} H_{2}\right)\right]}\right] \\
& \times\left[\left(\epsilon_{1 n}-H_{i}\right) \sinh ^{-2}\left(\epsilon_{1 n} H_{i}\right)+\left(\frac{\epsilon_{1 n}^{2}+1}{\epsilon_{1 n}^{2}-1}\right) \operatorname{coth}\left(\epsilon_{1 n} H_{i}\right)\right] \\
& +\frac{\epsilon_{1 n}}{\left(\frac{\sqrt{2 R e_{1}}}{(1-A)}+\frac{\sqrt{2 R e_{2}}}{(1+A)}\right)}\left[\frac{\left[\operatorname{coth}\left(\epsilon_{1 n} H_{1}\right)+\operatorname{coth}\left(\epsilon_{1 n} H_{2}\right)\right]^{2}}{(1-A) \operatorname{coth}\left(\epsilon_{1 n} H_{1}\right)+(1+A) \operatorname{coth}\left(\epsilon_{1 n} H_{2}\right)}\right] \\
& +4 \epsilon_{1 n}^{2}\left[\frac{\frac{(1+A)}{R e_{2}}-\frac{(1-A)}{R e_{1}}}{\frac{(1-A)}{\sqrt{R e_{1}}}+\frac{(1+A)}{\sqrt{R e_{2}}}}\right]\left[\frac{\frac{(1+A)}{\sqrt{R e_{2}}} \operatorname{coth}\left(\epsilon_{1 n} H_{2}\right)-\frac{(1-A)}{\sqrt{R e_{1}}} \operatorname{coth}\left(\epsilon_{1 n} H_{1}\right)}{(1-A) \operatorname{coth}\left(\epsilon_{1 n} H_{1}\right)+(1+A) \operatorname{coth}\left(\epsilon_{1 n} H_{2}\right)}\right] .
\end{aligned}
$$

\section{Appendix B. Linking the damped solution to existing inviscid theory}

We want to prove in this section that our damped two-layer wave solution

$$
\begin{aligned}
& \eta(r, \varphi, t)=\sum_{n=1}^{\infty} \frac{\left(\rho_{2}-\rho_{1}\right) d_{s} R \Omega^{2}}{\left[\left(\rho_{2}-\rho_{1}\right) g+\left(\frac{\epsilon_{1 n}}{R}\right)^{2} \gamma\right]} \frac{J_{1}\left(\epsilon_{1 n} \frac{r}{R}\right)}{\left(\epsilon_{1 n}^{2}-1\right) J_{1}\left(\epsilon_{1 n}\right)} \\
& \quad \times\left[\frac{\omega_{1 n}^{2}\left(\omega_{1 n}^{2}-\Omega^{2}\right)}{\left(\omega_{1 n}^{2}-\Omega^{2}\right)^{2}+4 \lambda_{1 n}^{2} \Omega^{2}} \cos (\Omega t-\varphi)+\frac{2 \lambda_{1 n} \Omega \omega_{1 n}^{2}}{\left(\omega_{1 n}^{2}-\Omega^{2}\right)^{2}+4 \lambda_{1 n}^{2} \Omega^{2}} \sin (\Omega t-\varphi)\right]
\end{aligned}
$$

includes the existing inviscid one-layer solution

$$
\eta(r, \varphi, t)=\frac{d_{s} \Omega^{2}}{2 g} \cos (\Omega t-\varphi)\left[r+\sum_{n=1}^{\infty} \frac{2 R}{\left(\epsilon_{1 n}^{2}-1\right)} \frac{\Omega^{2}}{\left(\omega_{1 n}^{2}-\Omega^{2}\right)} \frac{J_{1}\left(\epsilon_{1 n} \frac{r}{R}\right)}{J_{1}\left(\epsilon_{1 n}\right)}\right]
$$


derived by Reclari et al. (2014) as a limiting case. First, we consider the one-layer limit $\left(\rho_{1}=0\right)$ of (B 1) and neglect both damping $\left(\lambda_{1 n}=0\right)$ and surface tension $\gamma=0$. We find the symmetric solution

$$
\eta(r, \varphi, t)=\sum_{n=1}^{\infty} \frac{d_{s} R \Omega^{2}}{g} \frac{\omega_{1 n}^{2}}{\left(\omega_{1 n}^{2}-\Omega^{2}\right)} \frac{J_{1}\left(\epsilon_{1 n} \frac{r}{R}\right)}{\left(\epsilon_{1 n}^{2}-1\right) J_{1}\left(\epsilon_{1 n}\right)} \cos (\Omega t-\varphi) .
$$

Then, we can apply the transformation

$$
\frac{\omega_{1 n}^{2}}{\omega_{1 n}^{2}-\Omega^{2}}=1+\frac{\Omega^{2}}{\omega_{1 n}^{2}-\Omega^{2}}
$$

giving

$$
\eta(r, \varphi, t)=\frac{d_{s} \Omega^{2}}{2 g} \cos (\Omega t-\varphi) \sum_{n=1}^{\infty} \frac{J_{1}\left(\epsilon_{1 n} \frac{r}{R}\right)}{J_{1}\left(\epsilon_{1 n}\right)}\left[\frac{2 R}{\left(\epsilon_{1 n}^{2}-1\right)}+\frac{2 R}{\left(\epsilon_{1 n}^{2}-1\right)} \frac{\Omega^{2}}{\left(\omega_{1 n}^{2}-\Omega^{2}\right)}\right] .
$$

The first term of the sum is independent of the eigenfrequencies $\omega_{1 n}$ and can now be transformed using the Fourier-Bessel series (Reclari 2013)

$$
r=\sum_{n=1}^{\infty} \frac{2 R}{\left(\epsilon_{1 n}^{2}-1\right)} \frac{J_{1}\left(\frac{\epsilon_{1 n} r}{R}\right)}{J_{1}\left(\epsilon_{1 n}\right)}
$$

finally yielding the classical solution (B 1). This decomposition clarifies that the responding elevation of the free surface is always composed of a flat disc solution and the sum of radial free gravity wave modes. A consideration of non-vanishing surface tension $\gamma \neq 0$ inhibits such a decomposition since the surface tension force itself depends on the wave modes $\epsilon_{1 n}$. Surface tension can only act on curved surfaces such that the disc solution cannot exist in the capillary wave regime. It can be attributed to the balance between centrifugal acceleration and the conservative gravitational force alone.

The symmetric part of (B 1) can also be decomposed without neglecting linear damping forces when $\gamma=0$. Then the transformation

$$
\frac{\omega_{1 n}^{2}\left(\omega_{1 n}^{2}-\Omega^{2}\right)}{\left(\omega_{1 n}^{2}-\Omega^{2}\right)^{2}+4 \lambda_{1 n}^{2} \Omega^{2}}=1+\frac{\Omega^{2}\left(\omega_{1 n}^{2}-\Omega^{2}\right)-4 \lambda_{1 n}^{2} \Omega^{2}}{\left(\omega_{1 n}^{2}-\Omega^{2}\right)^{2}+4 \lambda_{1 n}^{2} \Omega^{2}}
$$

can be applied instead of (B 4) to separate the disc solution. This transformation yields the expression (2.32), which we have used in $\$ 2.4$ in order to prove that the discwave transition is independent of linear damping.

\section{REFERENCES}

Abramowitz, M. \& Stegun, I. A. 1972 Handbook of Mathematical Functions, 10th edn. vol. 55. National Bureau of Standards.

ABRAMSON, H. N. 1966 The dynamic behavior of liquids in moving containers, with applications to space vehicle technology. Tech. Rep. SP-106. NASA. 
Alpresa, P., Sherwin, S., Weinberg, P. \& Van Reeuwijk, M. 2018 a Orbitally shaken shallow fluid layers. I. Regime classification. Phys. Fluids 30, 032107.

Alpresa, P., Sherwin, S., Weinberg, P. \& Van Reeuwijk, M. $2018 b$ Orbitally shaken shallow fluid layers. II. An improved wall shear stress model. Phys. Fluids 30, 032108.

BAUER, H. F. \& EIDEL, W. 1997 Oscillations of a viscous liquid in a cylindrical container. Aerosp. Sci. Technol. 8, 519-532.

BAUER, H. F. \& EIDEL, W. 1999 Free oscillations and response of a viscous liquid in a rigid circular cylindrical tank. Aerosp. Sci. Technol. 3, 495-512.

Bojarevics, V. \& Romerio, M. V. 1994 Long waves instability of liquid metal-electroyte interface in aluminium electrolysis cell: a generalization of Sele's criterion. Eur. J. Mech. (B/Fluids) 13, 33-56.

Bouvard, J., Herreman, W. \& Moisy, F. 2017 Mean mass transport in an orbitally shaken cylindrical container. Phys. Rev. Fluids 2, 084801.

Büchs, J., Maier, U., Milbradt, C. \& Zoels, B. 2000 Power consumption in shaking flasks on rotary shaking machines: II. Nondimensional description of specific power consumption and flow regimes in unbaffled flasks at elevated liquid viscosity. Biotechnol. Bioengng. 68, 594-601.

CAse, K. M. \& PARKinson, W. C. 1956 Damping of surface waves in an incompressible liquid. J. Fluid Mech. 2 (2), 172-184.

Discacciati, M., Hacker, D., Quarteroni, A., Quinodoz, S., Tissot, S. \& Wurm, F. M. 2013 Numerical simulation of orbitally shaken viscous fluids with free surface. Intl J. Numer. Meth. Fluids 71, 294-315.

Ducci, A. \& WeheliYe, H. 2014 Orbitally shaken bioreactorsviscosity effects on flow characteristics. AIChE J. 60, 3951-3968.

Eckert, M. 2019 Ludwig Prandtl - A Life for Fluid Mechanics and Aeronautical Research. Springer.

Faltinsen, O. M., Lukovsky, I. A. \& TiмокнA, A. N. 2016 Resonant sloshing in an upright annular tank. J. Fluid Mech. 804, 608-645.

Faltinsen, O. M. \& Timokha, A. N. 2009 Sloshing. Cambridge University Press.

Faltinsen, O. M. \& TimoKhA, A. N. 2019 An inviscid analysis of the prandtl azimuthalmass transport during swirl-type sloshing. J. Fluid Mech. 865, 884-903.

Filipovic, N., Ghimire, K., Saveljic, I., Milosevic, Z. \& RuegG, C. 2016 Computational modeling of shear forces and experimental validation of endothelial cell responses in an orbital well shaker system. Comput. Meth. Biomech. Biomed. Engng 19 (6), 581-590.

Henderson, D. M. \& Miles, J. W. 1994 Surface-wave damping in a circular cylinder with a fixed contact line. J. Fluid Mech. 275, 285-299.

Herreman, W., Nore, C., Guermond, J.-L., Cappanera, L., Weber, N. \& Horstmann, G. M. 2019 Perturbation theory for metal pad roll instability in cylindrical reduction cells. J. Fluid Mech. 878, 598-646.

Horstmann, G. M., Weber, N. \& Weier, T. 2018 Coupling and stability of interfacial waves in liquid metal batteries. J. Fluid Mech. 845, 1-35.

Horstmann, G. M., Wylega, M. \& Weier, T. 2019 Measurement of interfacial wave dynamics in orbitally shaken cylindrical containers using ultrasound pulse-echo techniques. Exp. Fluids 60, 56.

Hutton, R. E. 1964 Fluid-particle motion during rotary sloshing. Trans. ASME J. Appl. Mech. 31, 123.

Ibrahim, R. A. 2005 Liquid Sloshing Dynamics Theory and Applications. Cambridge University Press.

Kelley, D. \& WeIER, T. 2018 Fluid mechanics of liquid metal batteries. Appl. Mech. Rev. 70 (2), 020801.

Kim, H. M. \& Kizito, J. P. 2009 Stirring free surface flows due to horizontal circulatory oscillation of partially filled container. Chem. Engng Commun. 196, 1300-1321.

KLÖCKNER, W. \& BÜCHS, J. 2012 Advances in shaking technologies. Trends Biotech. 30 (6), $307-314$. 
Klöckner, W., Diederichs, S. \& Büchs, J. 2014 Orbitally shaken single-use bioreactors. Adv. Biochem. Engng Biotechnol. 138, 45-60.

Klöckner, W., Tissot, S., Wurm, F. \& BÜchs, J. 2012 Power input correlation to characterize the hydrodynamics of cylindrical orbitally shaken bioreactors. Biochem. Engng J. 65, 63-69.

Kochin, N. E., Kibel, I. A.\& Roze, N. V. 1964 Theoretical Hydromechanics. Wiley.

Micheletti, M., Barrett, T., Doig, S. D., Baganz, F., Levy, M. S., Woodley, J. M. \& Lye, G. J. 2006 Fluid mixing in shaken bioreactors: Implications for scale-up predictions from microlitre-scale microbial and mammalian cell cultures. Chem. Engng Sci. 61 (9), 2939-2949.

Miles, J. W. 1991 The capillary boundary layer for standing waves. J. Fluid Mech. 222, 197-205.

Miles, J. W. \& Henderson, D. M. 1998 A note on interior vs. boundary-layer damping of surface waves in a circular cylinder. J. Fluid Mech. 364, 319-323.

Moisy, F., Bouvard, J. \& HerReman, W. 2018 Counter-rotation in an orbitally shaken glass of beer. Europhys. Lett. 122, 34002.

Pieralisi, I., Rodriguez, G., Micheletti, M., Paglianti, A. \& Ducci, A. 2016 Microcarriers suspension and flow dynamics in orbitally shaken bioreactors. Chem. Engng Res. Des. 108, 198-209.

PrandtL, L. 1949 Erzeugung von Zirkulationen beim Schütteln von Gefäßen. Z. Angew. Math. Mech. 29, 8-9.

RAYNOVSKYY, I. \& Timokha, A. $2018 a$ Damped steady-state resonant sloshing in a circular base container. Fluid Dyn. Res. 50, 045502.

RAYNovskyy, I. \& TImokha, A. $2018 b$ Steady-state resonant sloshing in an upright cylindrical container performing a circular orbital motion. Math. Probl. Engng 2018, 5487178.

ReClari, M. 2013 Hydrodynamics of orbital shaken bioreactors. PhD thesis, École Polytechnique Fédérale De Lausanne.

Reclari, M., Dreyer, M., Tissot, S., Obreschiow, D., Wurm, F. M. \& Farhat, M. 2014 Surface wave dynamics in orbital shaken cylindrical containers. Phys. Fluids 26, 052104.

Rodriguez, G., Anderlei, T., Micheletti, M., Yianneskis, M. \& Ducci, A. 2014 On the measurement and scaling of mixing time in orbitally shaken bioreactors. Biochem. Engng J. 82, $10-21$.

Rodriguez, G., Micheletti, M. \& DucCI, A. 2018 Macro- and micro-scale mixing in a shaken bioreactor for fluids of high viscosity. Chem. Engng Res. Des. 132, 890-901.

Rodriguez, G., Weheliye, W., Anderlei, T., Micheletti, M., Yianneskis, M. \& Ducci, A. 2013 Mixing time and kinetic energy measurements in a shaken cylindrical bioreactor. Chem. Engng Res. Des. 91 (11), 2084-2097.

Thomas, J. M. D., Chakraborty, A., Berson, R. E., Shakeri, M. \& Sharp, M. K. 2017 Validation of a CFD model of an orbiting culture dish with PIV and analytical solutions. AIChE J. 63, 4233-4242.

TImokha, A. N. \& RAYNovskyY, I. A. 2017 The damped sloshing in an upright circular tank due to an orbital forcing. Dopov. Nats. Akad. Mauk Ukr. 10, 48-53.

Tissot, S., Farhat, M., Hacker, D. L., Anderlei, T., Kühner, M., Comninellis, C. \& WURM, F. 2010 Determination of a scale-up factor from mixing time studies in orbitally shaken bioreactors. Biochem. Engng J. 52, 181-186.

Tucs, A., Bojarevics, V. \& Pericleous, K. 2018 Magnetohydrodynamic stability of large scale liquid metal batteries. J. Fluid Mech. 852, 453-483.

Viola, F. \& Gallaire, F. 2018 Theoretical framework to analyze the combined effect of surface tension and viscosity on the damping rate of sloshing waves. Phys. Rev. Fluids 3, 094801.

Weber, N., Beckstein, P., Herreman, W., Horstmann, G. M., Nore, C., Stefani, F. \& WEIER, T. 2017 Sloshing instability and electrolyte layer rupture in liquid metal batteries. Phys. Fluids 29, 054101.

Weheliye, H., YiAnNeskis, M. \& DuCCI, A. 2013 On the fluid dynamics of shaken bioreactorsflow characterization and transition. AIChE J. 59, 334-344. 
Weheliye, W. H., Cagney, N., Rodriguez, G., Micheletti, M. \& Ducci, A. 2018 Mode decomposition and lagrangian structures of the flow dynamics in orbitally shaken bioreactors. Phys. Fluids 30, 033603.

Zhang, X., Bürki, C.-A., Stettler, M., De Sanctis, D., Perrone, M., Discacciati, M., Parolini, N., DeJesus, M., Hacker, D. L., Quarteroni, A. \& Wurm, F. 2009 Efficient oxygen transfer by surface aeration in shaken cylindrical containers for mammalian cell cultivation at volumetric scales up to 1000 1. Biochem. Engng J. 45, 41-47.

Zhu, L., HAN, W., Song, B. \& WANG, Z. 2018 Characterizing the fluid dynamics in the flow fields of cylindrical orbitally shaken bioreactors with different geometry sizes. Engng Life Sci. 18, 570-578. 